\title{
U.S. DEPARTMENT OF ENERGY PORTSMOUTH ANNUAL ENVIRONMENTAL REPORT DATA FOR 1995
}

Date Published: April 1997

Prepared by

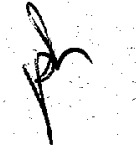

Environmental Compliance Division

Portsmouth Site

LOCKHEED MARTIN ENERGY SYSTEMS, INC.

P.O. Box 628

Piketon, Ohio 45661

for the

U.S. DEPARTMENT OF ENERGY

under contract no. DE-AC05-84OR21400

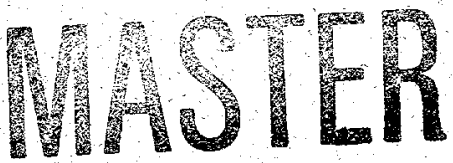





\section{Contents}

Page

List of Tables $\ldots \ldots \ldots \ldots \ldots \ldots \ldots \ldots \ldots \ldots \ldots \ldots \ldots \ldots \ldots$

Acronyms and Abbreviations $\ldots \ldots \ldots \ldots \ldots \ldots \ldots \ldots \ldots \ldots \ldots \ldots \ldots$

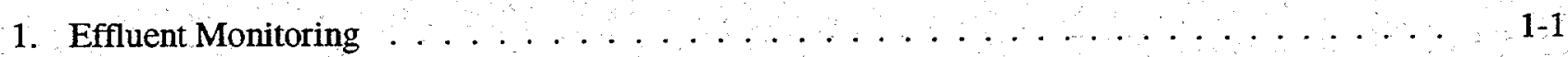

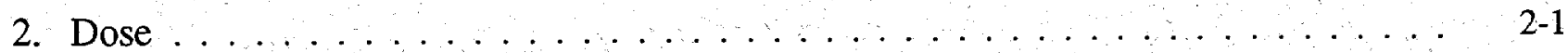

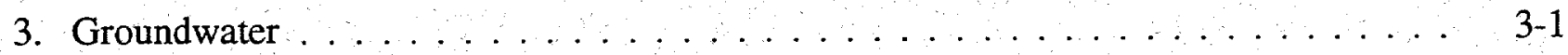





\section{DISCLAIMER}

This report was prepared as an account of work sponsored by an agency of the United States Government. Neither the United States Government nor any agency thereof, nor any of their employees, make any warranty, express or implied, or assumes any legal liability or responsibility for the accuracy, completeness, or usefulness of any information, apparatus, product, or process disclosed, or represents that its use would not infringe privately owned rights. Reference herein to any specific commercial product, process, or service by trade name, trademark, manufacturer, or otherwise does not necessarily constitute or imply its endorsement, recommendation, or favoring by the United States Government or any agency thereof. The views and opinions of authors expressed herein do not necessarily state or reflect those of the United States Government or any agency thereof. 


\section{DISCLAMMER}

Portions of this document may be illegible in electronic image products. Images are produced from the best available original document. 


\section{List of Tables}

Table

Page

1.1 Uranium concentrations in water samples for $1995 \ldots \ldots \ldots \ldots \ldots \ldots \ldots \ldots \ldots \ldots \ldots \ldots \ldots$

1.2 Radioactivity concentrations in water samples for $1995 \ldots \ldots \ldots \ldots \ldots \ldots \ldots \ldots$

1.3 DOE/PORTS NPDES permit summary for $1995 \ldots \ldots \ldots \ldots \ldots \ldots \ldots \ldots \ldots \ldots$

1.4 NPDES discharge and compliance rates for $1995 \ldots \ldots \ldots \ldots \ldots \ldots \ldots \ldots \ldots \ldots$

2.1 Committed effective dose rate conversion factors $\ldots \ldots \ldots \ldots \ldots \ldots \ldots \ldots \ldots \ldots$

2.2 Release point parameters and receptor locations used in 1995 dose calculations _ _ _ . . $\quad 2-3$

2.3 Activities (Ci), solubilities, and AMAD $(\mu \mathrm{m})$ of radionuclides released from

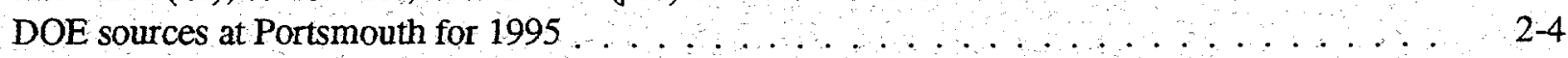

2.4 Predicted radiation doses from airborne releases at DOE/PORTS for $1995 \ldots \ldots \ldots$

3.1 DOE/PORTS groundwater monitoring for 1995: results for trichloroethene at X-701B _ . . $3-3$

3.2 DOE/PORTS groundwater monitoring for 1995: results for radionuclide parameters at $\mathrm{X}$-701B holding pond $\ldots \ldots \ldots \ldots \ldots \ldots \ldots \ldots \ldots \ldots \ldots \ldots$

3.3 DOR/PORTS groundwater monitoring for 1995: results for volatile organic compounds at X-749. .

3.4 DOE/PORTS groundwater monitoring for 1995: results for radionuclide parameters at X-7.49

3.5 DOE/PORTS groundwater monitoring for 1995: results for volatile organic compounds at $\mathrm{X}-231 \mathrm{~B} \ldots \ldots \ldots \ldots \ldots \ldots \ldots \ldots \ldots \ldots \ldots \ldots \ldots \ldots \ldots \ldots \ldots \ldots \ldots \ldots$

3.6 DOE/PORTS groundwater monitoring for 1995: results for radionuclide parameters at $\mathrm{X}-231 \mathrm{~B} \ldots \ldots \ldots \ldots \ldots \ldots \ldots \ldots \ldots \ldots \ldots \ldots \ldots \ldots \ldots \ldots \ldots \ldots \ldots \ldots$

3.7 DOE/PORTS groundwater monitoring for 1995: results for volatile organic compounds at $\mathrm{X}-616$

3.8 DOE/PORTS groundwater monitoring for 1995: results for dissolved and total chromium at $\mathrm{X}-616$. 
3.9 DOE/PORTS groundwater monitoring for 1995: results for radionuclide parameters at surface water monitoring locations

3.10 DOE/PORTS groundwater monitoring for 1995: results for volatile organic compounds at surface water monitoring locations $\ldots \ldots \ldots \ldots \ldots \ldots \ldots \ldots \ldots \ldots \ldots \ldots \ldots \ldots$

3.11 DOE/PORTS groundwater monitoring for 1995: results for trichloroethene at X-701C . . . .

3.12 DOE/PORTS groundwater monitoring for 1995: results for radionuclide parameters at $\mathrm{X}-701 \mathrm{C}$

3.13 DOE/PORTS groundwater monitoring for 1995: results for radionuclide parameters at $\mathrm{X}-735$

3.14. DOE/PORTS groundwater monitoring for 1995: results for trichloroethene at X-735 


\section{Acronyms and Abbreviations}

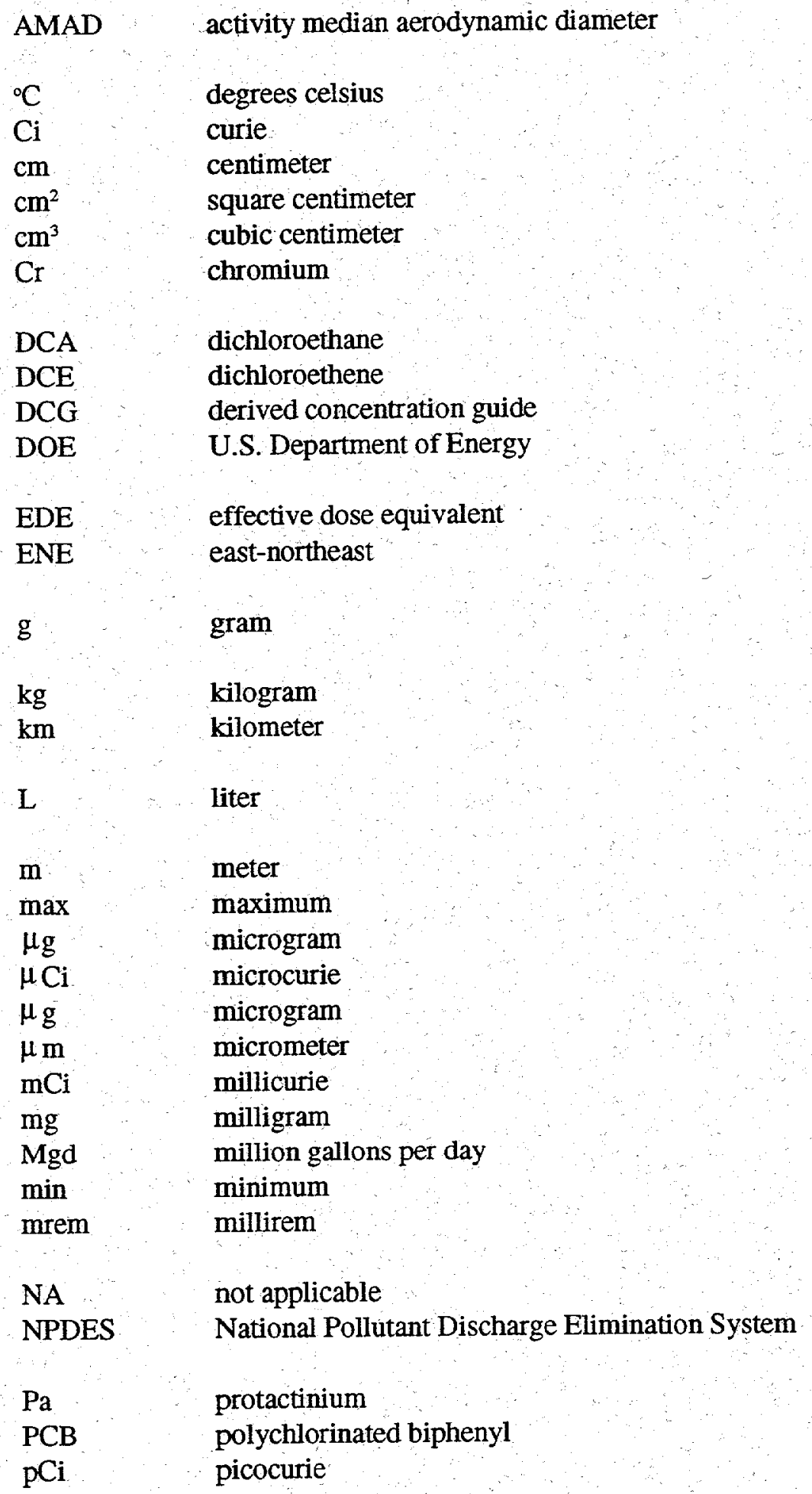


$s$

SU

Tc

TCA

TCE

Th

U

USEC

second

standard unit

technetium

trichloroethane

trichloroethene

thorium

uranium

United States Enrichment Corporation 


\section{Introduction}

Environmental monitoring on the Portsmouth Gaseous Diffusion Plant site is conducted throughout the year. Monitoring ensures that the site is a safe place to work, that plant operations do not adversely affect neighboring communities, and that activities comply with federal and state regulations.

This document is a compilation of the environmental monitoring data for calendar year 1995 and is intended as a tool for analysts in environmental monitoring, environmental restoration, and other related disciplines. The data in this document form the basis for the summary information in the Portsmouth Annual Environmental Report for 1995 (POEF-LMES-85). 
1. Effluent Monitoring 
Table 1.1. Uranium concentrations in water samples for 1995

\begin{tabular}{cccccc}
\hline & & \multicolumn{5}{c}{ Concentration } & \\
\cline { 3 - 6 } $\begin{array}{c}\text { NPDES } \\
\text { outfall }\end{array}$ & $\begin{array}{c}\text { Number of } \\
\text { samples }\end{array}$ & $\begin{array}{c}\text { Maximum } \\
(\mathrm{mg} / \mathrm{L})\end{array}$ & $\begin{array}{c}\text { Minimum } \\
(\mathrm{mg} / \mathrm{L})\end{array}$ & $\begin{array}{c}\text { Average } \\
(\mathrm{mg} / \mathrm{L})\end{array}$ & $\begin{array}{c}\text { Standard } \\
\text { deviation }\end{array}$ \\
\hline 012 & 12 & $<0.002$ & $<0.001$ & $<0.001$ & 0.25 \\
013 & 12 & $<0.002$ & $<0.001$ & $<0.001$ & 0.28 \\
\hline
\end{tabular}

${ }^{a}$ National Pollutant Discharge Elimination System.

Table 1.2. Radioactivity concentrations in water samples for 1995

\begin{tabular}{|c|c|c|c|c|c|c|}
\hline \multirow{3}{*}{$\begin{array}{l}\text { NPDES }^{a} \\
\text { outfall }\end{array}$} & \multirow{3}{*}{$\begin{array}{l}\text { Number of } \\
\text { samples }\end{array}$} & \multicolumn{5}{|c|}{ Concentration } \\
\hline & & \multirow{2}{*}{$\begin{array}{l}\text { Maximum } \\
(\mathrm{pCi} / \mathrm{L})\end{array}$} & \multirow{2}{*}{$\begin{array}{c}\text { Minimum } \\
(\mathrm{pCi} / \mathrm{L})\end{array}$} & \multicolumn{2}{|c|}{ Average } & \multirow{2}{*}{$\begin{array}{l}\text { Standard } \\
\text { deviation }\end{array}$} \\
\hline & & & & $(\mathrm{pCi} / \mathrm{L})$ & $\left(\% \mathrm{DCG}^{b}\right)$ & \\
\hline & & & Gross alpha & & & \\
\hline 012 & $12(12)^{c}$ & $<6$ & $<3$ & $<4.4$ & 0.98 & 0.759 \\
\hline 013 & $12(12)^{c}$ & $<12$ & $<4$ & $<6.2$ & 1.14 & 2.511 \\
\hline 010 & 17 & $<24$ & $<22$ & $<225$ & 022 & 0.866 \\
\hline 012 & & 24 & 20 & $<23$ & 022 & 0.745 \\
\hline & & 24 & Gross beta & & & \\
\hline 012 & 12 & $<11$ & $<8$ & $<9.2$ & $\mathrm{NA}^{d}$ & 1.344 \\
\hline 013 & 12 & $<11$ & $<8$ & $<9.0$ & NA & 1.291 \\
\hline
\end{tabular}

'National Pollutant Discharge Elimination System.

${ }^{b}$ Derived concentration guide.

Number in parentheses indicates the number of samples that were below the detection limit for gross alpha activity.

${ }^{d}$ Not applicable. 
Table 1.3. DOE/PORTS NPDES ${ }^{a}$ permit summary for 1995

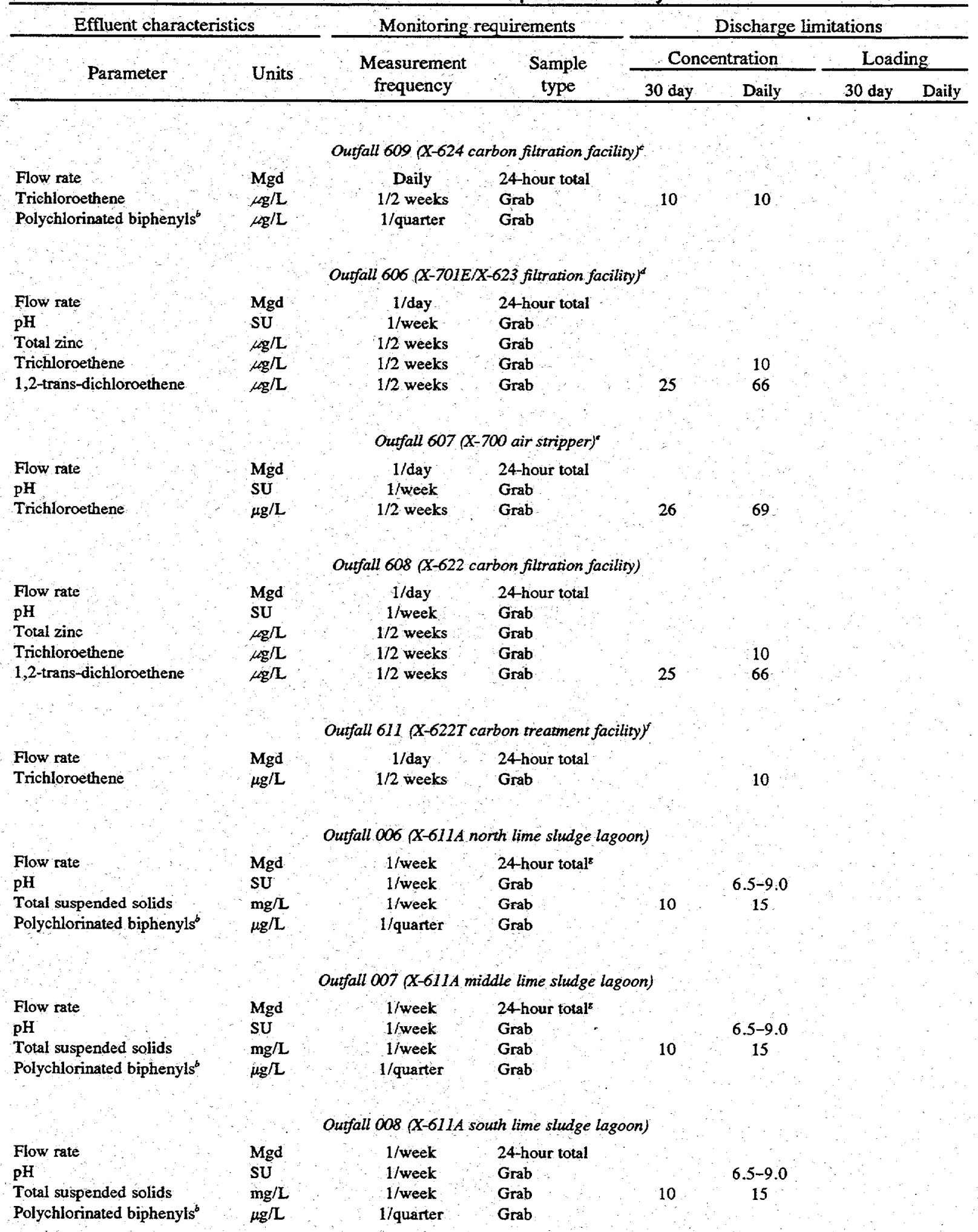


Table 1.3. DOE/PORTS NPDES ${ }^{a}$ permit summary for 1995 (continued)

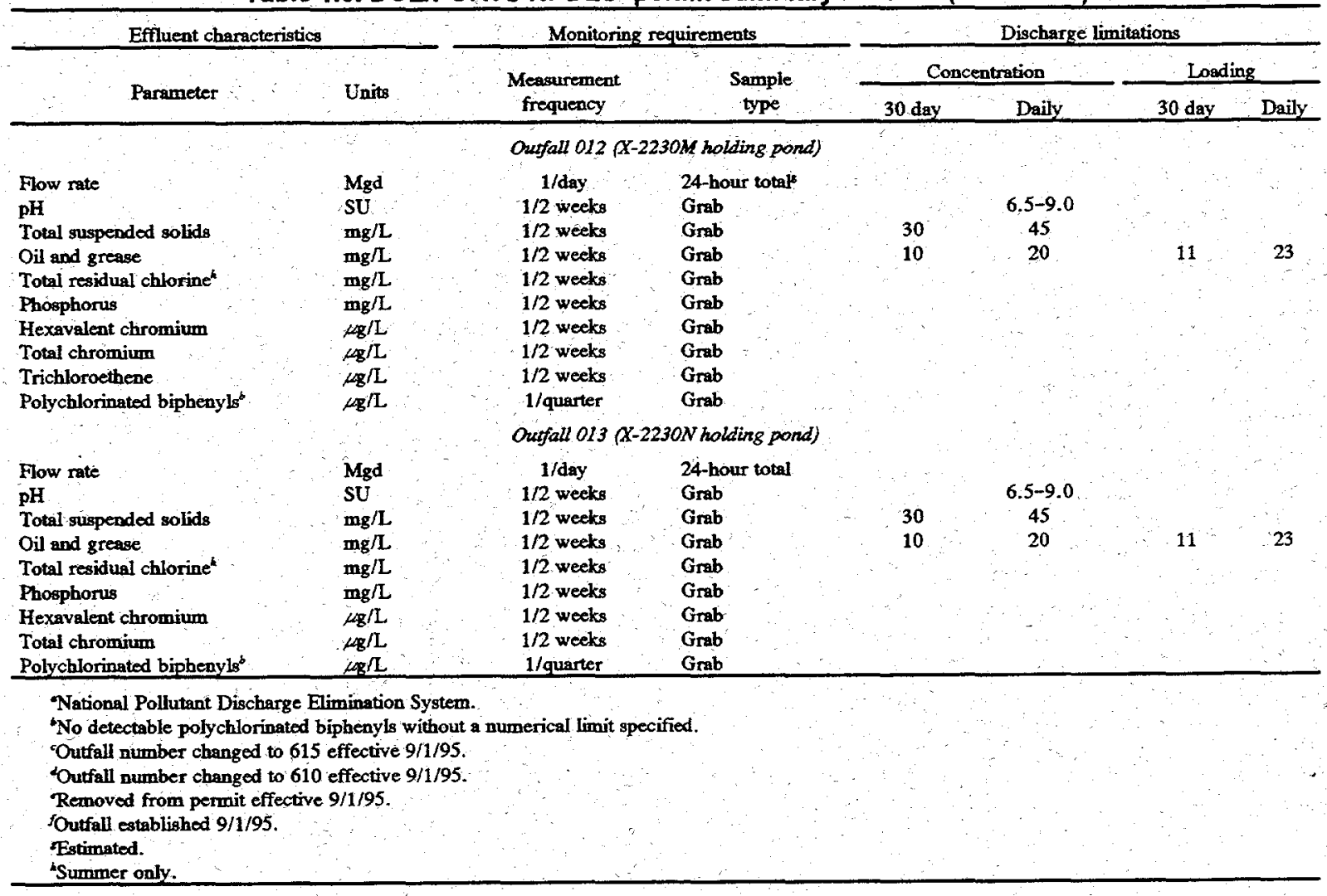


Table 1.4. NPDES ${ }^{\mathrm{a}}$ discharge and compliance rates for 1995

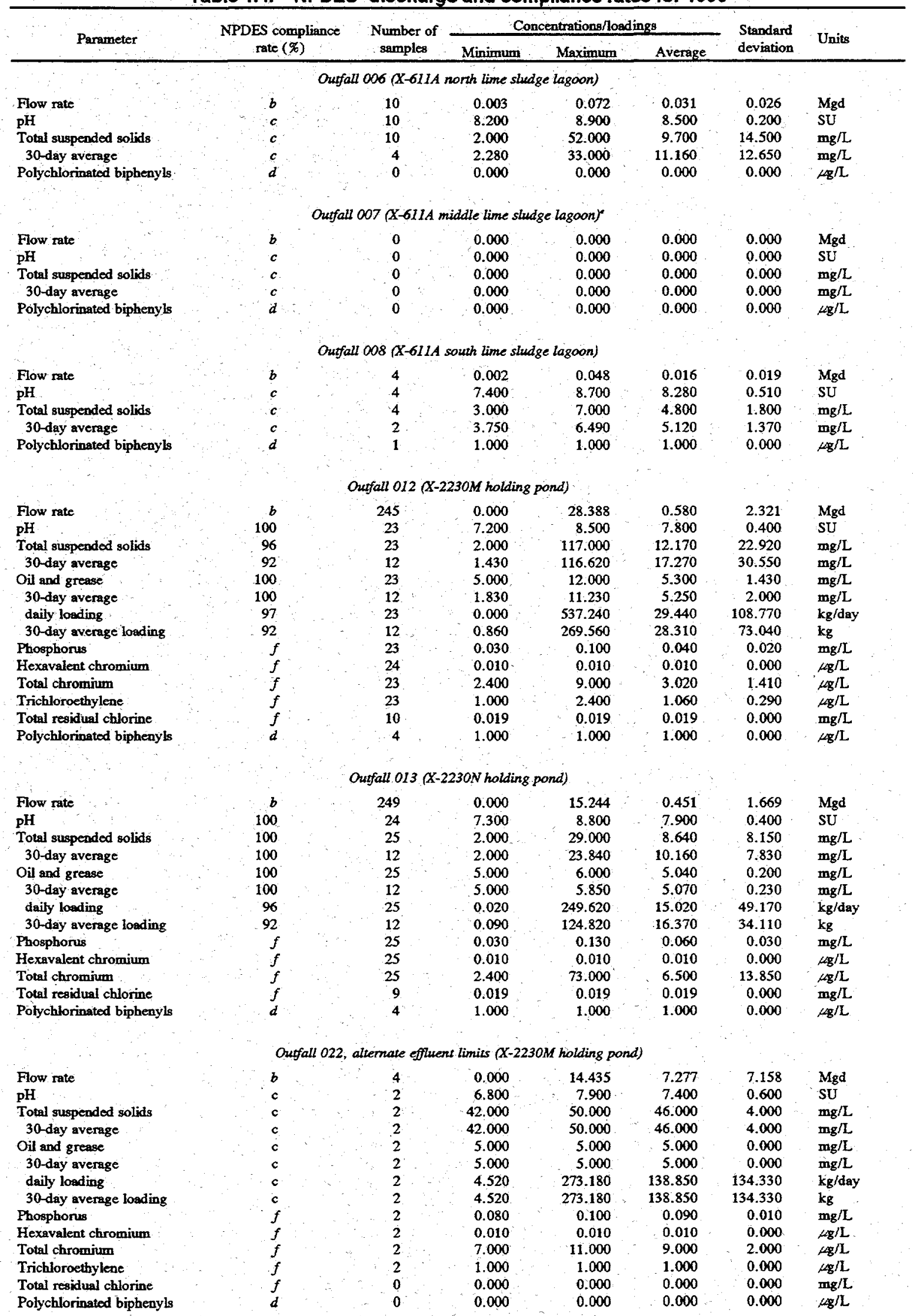


Táble 1.4. NPDES ${ }^{a}$ discharge and compliance rates for 1995 (continued)

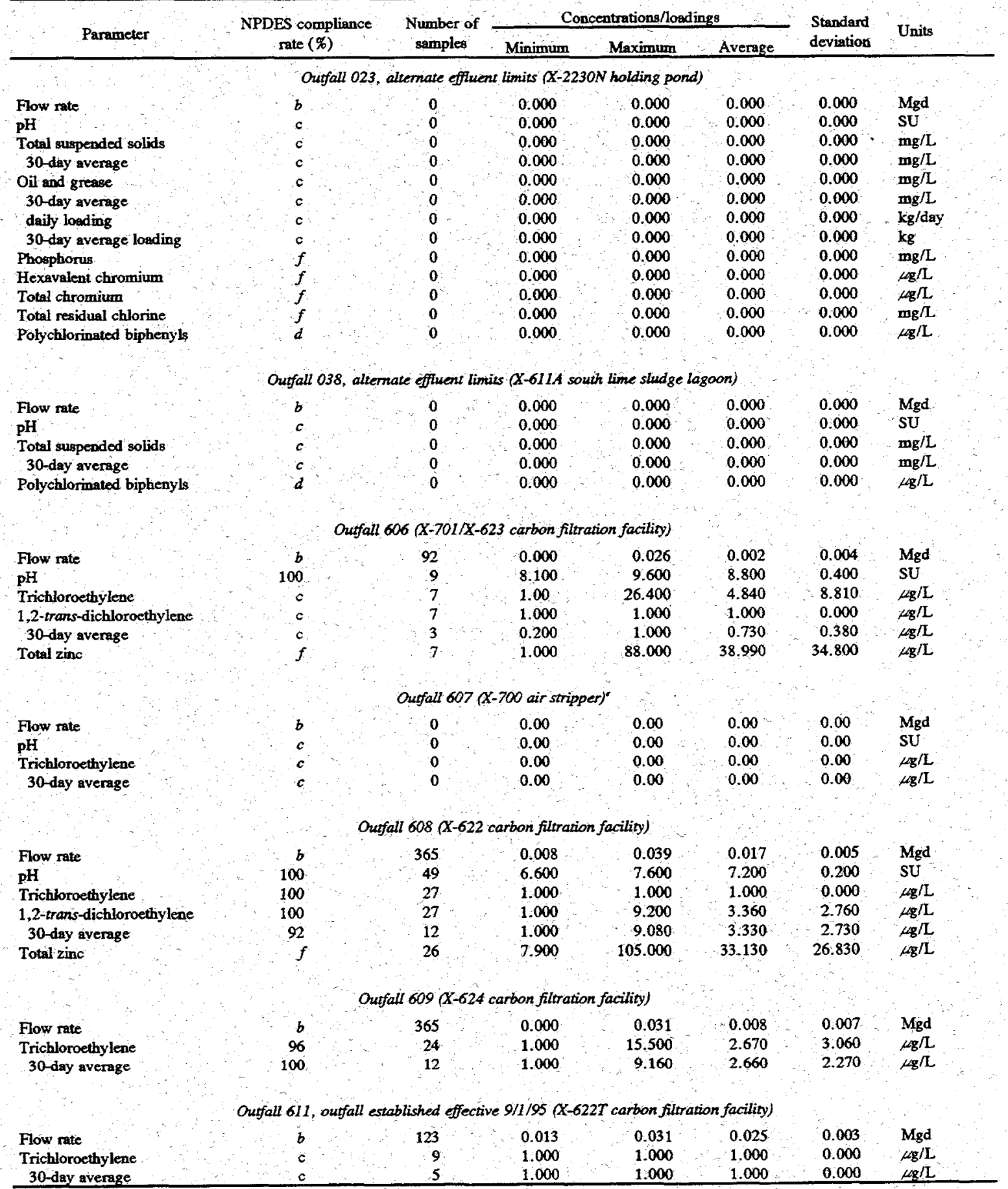

National Pollutant Discharge Elimination System.

${ }^{b}$ Flow does not have a numerical limit, no compliance rates are generated.

Insuffiently frequent flow to calculate useful compliance rate.

The permit specifies no detectable polychlorinated biphenyls in the effluent without setting a numerical limit of detection.

No flow throughout entire calendar year.

Monitoring only required. 
2. Dose 
Table 2.1. Committed effective dose rate conversion factors ${ }^{a}$

\begin{tabular}{lcccc}
\hline $\begin{array}{c}\text { Radionuclide } \\
\text { (solubility) }\end{array}$ & $\begin{array}{c}\text { Inhalation } \\
(\mathrm{rem} / \mu \mathrm{Ci})\end{array}$ & $\begin{array}{c}\text { Ingestion } \\
(\mathrm{rem} / \mu \mathrm{Ci})\end{array}$ & $\begin{array}{c}\text { Immersion } \\
(\text { mrem/year } \\
\left.\text { per } \mu \mathrm{Ci} / \mathrm{cm}^{3}\right)\end{array}$ & $\begin{array}{c}\text { Ground surfaces } \\
(\mathrm{mrem} / \mathrm{year} \\
\left.\text { per } \mu \mathrm{Ci} / \mathrm{cm}^{2}\right)\end{array}$ \\
\hline${ }^{99} \mathrm{Tc}$ (D) ${ }^{b}$ & $1.03 \times 10^{-3}$ & $1.45 \times 10^{-3}$ & $2.55 \times 10^{3}$ & $6.03 \times 10^{-1}$ \\
${ }^{234} \mathrm{U}$ (D) & 2.67 & $2.74 \times 10^{-1}$ & $7.46 \times 10^{5}$ & $8.00 \times 10^{2}$ \\
${ }^{235} \mathrm{U}$ (D) & 2.54 & $2.64 \times 10^{-1}$ & $7.51 \times 10^{8}$ & $1.67 \times 10^{5}$ \\
${ }^{236} \mathrm{U}$ (D) & 2.53 & $2.60 \times 10^{-1}$ & $5.90 \times 10^{5}$ & $7.27 \times 10^{2}$ \\
${ }^{238} \mathrm{U}$ (D) & 2.40 & $2.47 \times 10^{-1}$ & $5.06 \times 10^{5}$ & $6.41 \times 10^{2}$ \\
${ }^{231} \mathrm{Th}(\mathrm{W})^{b}$ & $9.62 \times 10^{-4}$ & $1.35 \times 10^{-3}$ & $5.82 \times 10^{1}$ & 1.91 \\
${ }^{234} \mathrm{Th}$ (W) & $3.06 \times 10^{-2}$ & $1.37 \times 10^{-2}$ & $3.73 \times 10^{7}$ & $9.71 \times 10^{3}$ \\
${ }^{234 m \mathrm{~Pa}}$ (W) & $3.77 \times 10^{-6}$ & $5.90 \times 10^{-6}$ & $5.93 \times 10^{7}$ & $1.12 \times 10^{4}$ \\
\hline
\end{tabular}

aFactors taken from the ALLRAD88 data file provided with CAP-88.

$b \mathrm{D}=$ soluble in blood; $\mathrm{W}=$ moderately soluble in blood.

Table 2.2. Release point parameters and receptor locations used in 1995 dose calculations

\begin{tabular}{llccccc}
\hline Name & Type & $\begin{array}{c}\text { Release } \\
\text { height } \\
(\mathrm{m})\end{array}$ & $\begin{array}{c}\text { Inner } \\
\text { diameter } \\
(\mathrm{m})\end{array}$ & $\begin{array}{c}\text { Gas exit } \\
\text { velocity } \\
(\mathrm{m} / \mathrm{s})\end{array}$ & $\begin{array}{c}\text { Gas exit } \\
\text { tempera } \\
\text { ture } \\
\left({ }^{\circ} \mathrm{C}\right)\end{array}$ & $\begin{array}{c}\text { Distance }(\mathrm{m}) \\
\text { and direction to } \\
\text { receptor }\end{array}$ \\
\hline Stack 1 & Point & 50 & 0.25 & 18 & Ambient & $1370 \mathrm{SE}$ \\
Stack 2 & Point & 20 & 0.97 & 24 & Ambient & $1530 \mathrm{E}$ \\
Stack $3^{b}$ & Point & 3 & 0.1 & 13.2 & Ambient & $1410 \mathrm{ESE}$ \\
Stack $4^{b}$ & Point & 20 & 0.2 & 30.5 & Ambient & $1450 \mathrm{ESE}$ \\
\hline
\end{tabular}

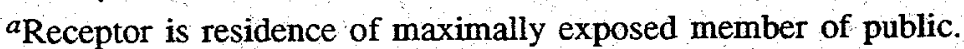

bDOE source. 
Table 2.3. Activities (Ci), solubilities, and $\mathrm{AMAD}^{a}(\mu \mathrm{m})$ of radionuclides released from DOE Sources at Portsmouth for 1995

\begin{tabular}{|c|c|c|c|c|c|}
\hline $\begin{array}{l}\text { Radionuclide } \\
\text { (solubility) }\end{array}$ & $\begin{array}{c}\mathrm{AMAD}^{a} \\
(\mu \mathrm{m})\end{array}$ & $\begin{array}{c}\text { Stack 1 } \\
\text { (USEC-1)b } \\
\text { Activity } \\
\text { (Ci) }\end{array}$ & $\begin{array}{c}\text { Stack } 2 \\
\text { (USEC-2)b } \\
\text { Activity } \\
\text { (Ci) }\end{array}$ & $\begin{array}{c}\text { Stack } 3 \\
\text { (DOE-1) } \\
\text { Activity } \\
\text { (Ci) }\end{array}$ & $\begin{array}{c}\text { Stack } 4 \\
\text { (DOE-2) } \\
\text { Activity } \\
\text { (Ci) }\end{array}$ \\
\hline${ }^{99} \mathrm{Tc}(\mathrm{D})^{\mathrm{c}}$ & 1 & $1.298 \mathrm{E}-2$ & $4.809 \mathrm{E}-4$ & $1.198 \mathrm{E}-3$ & $3.422 \mathrm{E}-5$ \\
\hline${ }^{234} \mathrm{U}$ (D) & 1 & $8.651 \mathrm{E}-3$ & $1.686 \mathrm{E}-5$ & $1.285 \mathrm{E}-4$ & $1.130 \mathrm{E}-5$ \\
\hline${ }^{235} \mathrm{U}$ (D) & 1 & $7.301 \mathrm{E}-4$ & $4.43 \mathrm{E}-7$ & $3.480 \mathrm{E}-6$ & $2.562 \mathrm{E}-7$ \\
\hline${ }^{236} \mathrm{U}$ (D) & 1 & $8.68 \mathrm{E}-7$ & $1.206 \mathrm{E}-9$ & $1.100 \mathrm{E}-8$ & 6.917 E-10 \\
\hline${ }^{238} \mathrm{U}(\mathrm{D})$ & 1 & $1.889 \mathrm{E}-4$ & $5.49 \mathrm{E}-7$ & $2.044 \mathrm{E}-6$ & $2.598 \mathrm{E}-8$ \\
\hline${ }^{231} \mathrm{Th}(\mathrm{W})^{\mathrm{c}}$ & 1 & $2.301 \mathrm{E}-4$ & $4.47 \mathrm{E}-7$ & $3.480 \mathrm{E}-6$ & $2.562 \mathrm{E}-7$ \\
\hline${ }^{234} \mathrm{Th}(\mathrm{W})$ & 1 & $1.888 \mathrm{E}-4$ & $5.48 \mathrm{E}-7$ & $2.044 \mathrm{E}-6$ & $2.598 \mathrm{E}-8$ \\
\hline${ }^{234 m} \mathrm{~Pa}(\mathrm{~W})$ & 1 & $1.888 \mathrm{E}-4$ & $5.48 \mathrm{E}-7$ & $2.044 \mathrm{E}-6$ & $2.598 \mathrm{E}-8$ \\
\hline
\end{tabular}

${ }^{a}$ Activity median aerodynamic diameter.

$b \mathrm{DOE}$ fraction only

$c \mathrm{D}=$ soluble in blood; $\mathrm{W}=$ moderately soluble in blood .

Table 2.4. Predicted radiation doses from airborne releases at DOE/PORTS for 1995

Effective dose equivalent (EDE) to:

$\mathrm{EDE}$

Maximum exposed individual ${ }^{a}$

$0.13 \mathrm{mrem}$

Population ${ }^{b}$

1.2 person-rem

Nearest community ${ }^{c}$

0.02 person-rem

${ }^{a}$ Maximum exposed individual resides on the reservation boundary, $1770 \mathrm{~m}(5807 \mathrm{ft})$ ENE of plant center.

${ }^{b}$ Collective EDE to general population within $80 \mathrm{~km}$ (50 miles) of plant site.

${ }^{c}$ Collective EDE to residents of nearest community (Piketon, Ohio). 
3. Groundwater 
Table 3.1. DOE/PORTS groundwater monitoring for 1995: results for trichloroethene at X-701B

\begin{tabular}{|c|c|c|c|c|c|c|}
\hline Well & Parameter & Min & Max & Mean & $\begin{array}{l}\text { Standard } \\
\text { Deviation }\end{array}$ & Units \\
\hline \multicolumn{7}{|c|}{ Berea Wells } \\
\hline X701-50B & Trichloroethene & $2.00 \mathrm{U}$ & $2.00 \mathrm{U}$ & & & $\mu \mathrm{g} / \mathrm{L}$ \\
\hline X701-58B & Trichloroethene & $2.00 \mathrm{U}$ & $2.00 \mathrm{U}$ & & & $\mu \mathrm{g} / \mathrm{L}$ \\
\hline X701-61B & Trichloroethene & $2.00 \mathrm{U}$ & $2.00 \mathrm{U}$ & & & $\mu \mathrm{g} / \mathrm{L}$ \\
\hline \multicolumn{7}{|c|}{ Gallia Wells } \\
\hline LBC-PZO3 & Trichloroethene & 370.00 & 820.00 & 595.00 & 318.20 & $\mu \mathrm{g} / \mathrm{L}$ \\
\hline LBC-PZO6 & Trichloroethene & $2.00 \mathrm{U}$ & $2.00 \mathrm{U}$ & & & $\mu \mathrm{g} / \mathrm{L}$ \\
\hline$X 701-02 G$ & Trichloroethene & 3.00 & 5.00 & 4.00 & 0.82 & $\mu \mathrm{g} / \mathrm{L}$ \\
\hline$X 701-05 G$ & Trichloroethene & 5.00 & 8.00 & 7.25 & 1.50 & $\mu \mathrm{g} / \mathrm{L}$ \\
\hline$\times 701-06 \mathrm{G}$ & Trichloroethene & 110.00 & 120.00 & 117.50 & 5.00 & $\mu \mathrm{g} / \mathrm{L}$ \\
\hline X701-08G & Trichloroethene & 440000.00 & 550000.00 & 486666.67 & 56862.41 & $\mu \mathrm{g} / \mathrm{L}$ \\
\hline$X 701-10 G$ & Trichloroethene & 1900.00 & 2400.00 & 2150.00 & 208.17 & $\mu \mathrm{g} / \mathrm{L}$ \\
\hline$\times 701-14 G$ & Trichloroethene & 210000.00 & 480000.00 & 332500.0 & 00125000.00 & $\mu \mathrm{g} / \mathrm{L}$ \\
\hline$X 701-15 G$ & Trichloroethene & 4.00 & 11.00 & 7.25 & 3.30 & $\mu \mathrm{g} / \mathrm{L}$ \\
\hline$X 701-16 G$ & Trichloroethene & $2.00 \mathrm{U}$ & $2.00 \mathrm{U}$ & & & $\mu \mathrm{g} / \mathrm{L}$ \\
\hline $\mathrm{X} 701-19 \mathrm{G}$ & Trichloroethene & $2.00 \mathrm{U}$ & $2.00 \mathrm{U}$ & & & $\mu \mathrm{g} / \mathrm{L}$ \\
\hline$X 701-21 G$ & Trichloroethene & 32.00 & 47.00 & 37.00 & 7.07 & $\mu \mathrm{g} / \mathrm{L}$ \\
\hline$X 701-23 G$ & Trichloroethene & $2.00 \mathrm{U}$ & $2.00 \mathrm{U}$ & & & $\mu \mathrm{g} / \mathrm{L}$ \\
\hline$X 701-24 G$ & Trichloroethene & 30000.00 & 48000.00 & 42000.00 & 8485.28 & $\mu \mathrm{g} / \mathrm{L}$ \\
\hline$X 701-25 G$ & Trichloroethene & $2.00 \mathrm{U}$ & $7.00 \mathrm{U}$ & & & $\mu \mathrm{g} / \mathrm{L}$ \\
\hline X701-30G & Trichloroethene & 17.00 & 20.00 & 18.25 & 1.50 & $\mu \mathrm{g} / \mathrm{L}$ \\
\hline$X 701-31 G$ & Trichloroethene & $2.00 \mathrm{U}$ & $2.00 \mathrm{U}$ & & & $\mu \mathrm{g} / \mathrm{L}$ \\
\hline$X 701-32 G$ & Trichloroethene & $2.00 \mathrm{U}$ & $2.00 \mathrm{U}$ & & & $\mu \mathrm{g} / \mathrm{L}$ \\
\hline $\mathrm{X} 701-38 \mathrm{G}$ & Trichloroethene & $2.00 \mathrm{U}$ & 5.00 & & & $\mu \mathrm{g} / \mathrm{L}$ \\
\hline$X 701-48 \mathrm{G}$ & Trichloroethene & $2.00 \mathrm{U}$ & $4.00 \mathrm{U}$ & & & $\mu \mathrm{g} / \mathrm{L}$ \\
\hline$X 701-49 G$ & Trichloroethene & $2.00 \mathrm{U}$ & $2.00 \mathrm{U}$ & & & $\mu \mathrm{g} / \mathrm{L}$ \\
\hline X701-BW1G & Trichloroethene & $2.00 \mathrm{U}$ & $2.00 \mathrm{U}$ & & & $\mu \mathrm{g} / \mathrm{L}$ \\
\hline X701-BW4G & Trichloroethene & $2.00 \mathrm{U}$ & $2.00 \mathrm{U}$ & & & $\mu \mathrm{g} / \mathrm{L}$ \\
\hline
\end{tabular}

Data Qualifer (U)-Parameter not detected. Result is attainable detection limit for the sample.

Mean and standard deviation functions not calculated if at least $50 \%$ of the results for the parameter were below detection. 
Table 3.2. DOE/PORTS groundwater monitoring for 1995: results for radionuclide parameters at X-701B holding pond

\begin{tabular}{|c|c|c|c|c|c|c|}
\hline Well & Parameter & Min & Max & Mean & $\begin{array}{l}\text { Standard } \\
\text { Deviation }\end{array}$ & Units \\
\hline \multicolumn{7}{|c|}{ Berea Wells } \\
\hline \multirow[t]{4}{*}{ X701-50B } & Gross Alpha & $10.00 \mathrm{U}$ & 21.00 & 17.50 & 5.07 & $\mathrm{pCi} / \mathrm{L}$ \\
\hline & Gross Beta & 10.00 & 21.00 & 16.00 & 4.69 & $\mathrm{pCi} / \mathrm{L}$ \\
\hline & Technetium & $22.00 \mathrm{U}$ & $22.00 \mathrm{U}$ & & & $\mathrm{pCi} / \mathrm{L}$ \\
\hline & Uranium & $1.00 \mathrm{U}$ & 3.80 & 2.05 & 1.34 & $\mu \mathrm{g} / \mathrm{L}$ \\
\hline \multirow[t]{4}{*}{ X701-58B } & Gross Alpha & 14.00 & 17.00 & 15.50 & 2.12 & $\mathrm{pCi} / \mathrm{L}$ \\
\hline & Gross Beta & 17.00 & 21.00 & 19.00 & 2.83 & $\mathrm{pCi} / \mathrm{L}$ \\
\hline & Technetium & $22.00 \mathrm{U}$ & $22.00 \mathrm{U}$ & & & $\mathrm{pCi} / \mathrm{L}$ \\
\hline & Uranium & 1.80 & 2.80 & 2.30 & 0.71 & $\mu \mathrm{g} / \mathrm{L}$ \\
\hline \multirow[t]{4}{*}{ X701-61B } & Gross Alpha & $22.00 \mathrm{U}$ & 180.00 & & & $\mathrm{pC} / \mathrm{L}$ \\
\hline & Gross Beta & 22.00 & 126.00 & & & $\mathrm{pCi} / \mathrm{L}$ \\
\hline & Technetium & $22.00 \mathrm{U}$ & $22.00 \mathrm{U}$ & & & $\mathrm{pCi} / \mathrm{L}$ \\
\hline & Uranium & 1.70 & 1.80 & 1.71 & 0.03 & $\mu g / L$ \\
\hline \multicolumn{7}{|c|}{ Gallia Wells } \\
\hline \multirow[t]{4}{*}{ LBC-PZ03 } & Gross Alpha & $5.00 \mathrm{U}$ & 5.00 & & & $\mathrm{pCi} / \mathrm{L}$ \\
\hline & Gross Beta & $8.00 \mathrm{U}$ & $11.00 \mathrm{U}$ & & & $\mathrm{pCi} / \mathrm{L}$ \\
\hline & Technetium & $22.00 \mathrm{U}$ & $22.00 \mathrm{U}$ & & & $\mathrm{pCi} / \mathrm{L}$ \\
\hline & Uranium & $1.00 \mathrm{U}$ & $1.00 \mathrm{U}$ & & & $\mu \mathrm{g} / \mathrm{L}$ \\
\hline \multirow[t]{4}{*}{ LBC-PZ06 } & Gross Alpha & $4.00 \mathrm{U}$ & $5.00 \mathrm{U}$ & & & $\mathrm{pCi} / \mathrm{L}$ \\
\hline & Gross Beta & $8.00 \mathrm{U}$ & $11.00 \mathrm{U}$ & & & $\mathrm{pCi} / \mathrm{L}$ \\
\hline & Technetium & $22.00 \mathrm{U}$ & $22.00 \mathrm{U}$ & & & $\mathrm{pCi} / \mathrm{L}$ \\
\hline & Uranium & $1.00 \mathrm{U}$ & 4.90 & 2.15 & 1.85 & $\mu \mathrm{g} / \mathrm{L}$ \\
\hline \multirow[t]{4}{*}{$X 701-02 \mathrm{G}$} & Gross Alpha & $5.00 \mathrm{U}$ & 10.00 & & & $\mathrm{pCi} / \mathrm{L}$ \\
\hline & Gross Beta & $8.00 \mathrm{U}$ & $11.00 \mathrm{U}$ & & & $\mathrm{pCi} / \mathrm{L}$ \\
\hline & Technetium & $22.00 \mathrm{U}$ & $24.00 \mathrm{U}$ & & & $\mathrm{pCi} / \mathrm{L}$ \\
\hline & Uranium & $1.00 \mathrm{U}$ & $1.00 \mathrm{U}$ & & & $\mu \mathrm{g} / \mathrm{L}$ \\
\hline \multirow[t]{3}{*}{$\mathrm{X} 701-05 \mathrm{G}$} & Gross Alpha & $5.00 \mathrm{U}$ & 10.00 & & & $\mathrm{pCi} / \mathrm{L}$ \\
\hline & Gross Beta & $8.00 \mathrm{U}$ & 12.00 & & & $\mathrm{pCi} / \mathrm{L}$ \\
\hline & Technetium & $22.00 \mathrm{U}$ & $22.00 \mathrm{U}$ & & & $\mathrm{pCi} / \mathrm{L}$ \\
\hline
\end{tabular}


Table 3.2. DOEJPORT's groundwater monitoring for 1995: results for radionuclide parameters at $X-701 B$ holding pond (continued)

\begin{tabular}{|c|c|c|c|c|c|c|}
\hline Well & Parameter & Min & $\operatorname{Max}$ & Mean & $\begin{array}{l}\text { Standard } \\
\text { Deviation }\end{array}$ & Units \\
\hline \multicolumn{7}{|c|}{ Gallia Wells (cont) } \\
\hline$X 701-05 G$ & Uranium & $1.00 \mathrm{U}$ & $1.00 \mathrm{U}$ & & & $\mu \mathrm{g} / \mathrm{L}$ \\
\hline \multirow[t]{4}{*}{ X701-06G } & Gross Alpha & $1.00 \mathrm{U}$ & $6.00 \mathrm{U}$ & & & $\mathrm{pCi} / \mathrm{L}$ \\
\hline & Gross Beta & 21.00 & 51.00 & 37.25 & 12.66 & $\mathrm{pCi} / \mathrm{L}$ \\
\hline & Technetium & 28.00 & 56.00 & 44.25 & 14.06 & $\mathrm{pCi} / \mathrm{L}$ \\
\hline & Uranium & $1.00 \mathrm{U}$ & $1.00 \mathrm{U}$ & & & $\mu \mathrm{g} / \mathrm{L}$ \\
\hline \multirow[t]{4}{*}{ X701-08G } & Gross Alpha & $4.00 \mathrm{U}$ & $5.00 \mathrm{U}$ & & & $\mathrm{pCi} / \mathrm{L}$ \\
\hline & Gross Beta & 109.00 & 172.00 & 144.33 & 32.19 & $\mathrm{pCi} / \mathrm{L}$ \\
\hline & Technetium & 165.00 & 227.00 & 200.00 & 31.76 & $\mathrm{pCi} / \mathrm{L}$ \\
\hline & Uranium & $1.00 \mathrm{U}$ & $1.00 \mathrm{U}$ & & & $\mu \mathrm{g} / \mathrm{L}$ \\
\hline \multirow[t]{4}{*}{$\mathrm{X} 701-10 \mathrm{G}$} & Gross Alpha & $5.60 \mathrm{U}$ & $6.00 \mathrm{U}$ & & & pCill \\
\hline & Gross Beta & $8.00 \mathrm{U}$ & 31.00 & & & pCilL \\
\hline & Technetium & $22.00 \mathrm{U}$ & $22.00 \mathrm{U}$ & & & $\mathrm{pCi} / \mathrm{L}$ \\
\hline & Uranium & $1.00 \mathrm{U}$ & 1.80 & & & $\mu \mathrm{g} / \mathrm{L}$ \\
\hline \multirow[t]{4}{*}{$X 701-14 G$} & Gross Alpha & $5.00 \mathrm{U}$ & $6.00 \mathrm{U}$ & & & $\mathrm{pCi} / \mathrm{L}$ \\
\hline & Gross Beta & 134.00 & 142.00 & 136.75 & 3.59 & $\mathrm{pCi} / \mathrm{L}$ \\
\hline & Technetium & 165.00 & 189.00 & 177.75 & 13.05 & $\mathrm{pCi} / \mathrm{L}$ \\
\hline & Uranium & $0.44 \mathrm{U}$ & $1.00 \mathrm{U}$ & & & $\mu \mathrm{g} / \mathrm{L}$ \\
\hline \multirow[t]{4}{*}{$\times 701-15 G$} & Gross Alpha & $4.00 \mathrm{U}$ & $4.00 \mathrm{U}$ & & & $\mathrm{pCi} / \mathrm{L}$ \\
\hline & Gross Beta & $8.00 \mathrm{U}$ & 10.00 & & & $\mathrm{pCi} / \mathrm{L}$ \\
\hline & Technetium & $22.00 \mathrm{U}$ & $22.00 \mathrm{U}$ & & & $\mathrm{pCi} / \mathrm{L}$ \\
\hline & Uranium & $1.00 \mathrm{U}$ & $1.00 \mathrm{U}$ & & & $\mu \mathrm{g} / \mathrm{L}$ \\
\hline \multirow[t]{4}{*}{$X 701-16 G$} & Gross Alpha & $4.00 \mathrm{U}$ & 6.00 & & & $\mathrm{pCi} / \mathrm{L}$ \\
\hline & Gross Beta & $8.00 \mathrm{U}$ & $11.00 \mathrm{U}$ & & & $\mathrm{pCi} / \mathrm{L}$ \\
\hline & Technetium & $22.00 \mathrm{U}$ & $22.00 \mathrm{U}$ & & & $\mathrm{pCi} / \mathrm{L}$ \\
\hline & Uranium & $1.00 \mathrm{U}$ & 1.50 & & & $\mu \mathrm{g} / \mathrm{L}$ \\
\hline \multirow[t]{3}{*}{$\mathrm{X} 701-19 \mathrm{G}$} & Gross Alpha & $3.90 \mathrm{U}$ & $5.00 \mathrm{U}$ & & & pCilL \\
\hline & Gross Beta & $8.00 \mathrm{U}$ & $11.00 \mathrm{U}$ & & & pCiL \\
\hline & Technetium & $22.00 \mathrm{U}$ & $22.00 \mathrm{U}$ & & & $\mathrm{pCi} / \mathrm{L}$ \\
\hline
\end{tabular}


Table 3.2. DOE/PORTS groundwater monitoring for 1995:

results for radionuclide parameters at $X-701 B$ holding pond (continued)

\begin{tabular}{|c|c|c|c|c|c|c|}
\hline Well & Parameter & Min & $\operatorname{Max}$ & Mean & $\begin{array}{l}\text { Standard } \\
\text { Deviation }\end{array}$ & Units \\
\hline \multicolumn{7}{|c|}{ Gallia Wells (cont) } \\
\hline $\mathrm{X} 701-19 \mathrm{G}$ & Uranium & $1.00 \mathrm{U}$ & $1.00 \mathrm{U}$ & & & $\mu \mathrm{g} / \mathrm{L}$ \\
\hline \multirow[t]{4}{*}{$\mathrm{X} 701-21 \mathrm{G}$} & Gross Alpha & $8.00 \mathrm{U}$ & $8.00 \mathrm{U}$ & & & $\mathrm{pCi} / \mathrm{L}$ \\
\hline & Gross Beta & 20.00 & 32.00 & 26.00 & 5.89 & $\mathrm{pCi} / \mathrm{L}$ \\
\hline & Technetium & 34.00 & 58.00 & 45.75 & 11.15 & $\mathrm{pCi} / \mathrm{L}$ \\
\hline & Uranium & $1.00 \mathrm{U}$ & $1.00 \mathrm{U}$ & & & $\mu \mathrm{g} / \mathrm{L}$ \\
\hline \multirow[t]{4}{*}{$X 701-23 G$} & Gross Alpha & $4.00 \mathrm{U}$ & $5.00 \mathrm{U}$ & & & $\mathrm{pCi} / \mathrm{L}$ \\
\hline & Gross Beta & $8.00 \mathrm{U}$ & $11.00 \mathrm{U}$ & & & $\mathrm{pCi} / \mathrm{L}$ \\
\hline & Technetium & $22.00 \mathrm{U}$ & $22.00 \mathrm{U}$ & & & $\mathrm{pCi} / \mathrm{L}$ \\
\hline & Uranium & $1.00 \mathrm{U}$ & $1.00 \mathrm{U}$ & & & $\mu \mathrm{g} / \mathrm{L}$ \\
\hline \multirow[t]{4}{*}{$X 701-24 G$} & Gross Alpha & $4.00 \mathrm{U}$ & 53.00 & & & $\mathrm{pCi} / \mathrm{L}$ \\
\hline & Gross Beta & 20.00 & 93.00 & 40.50 & 35.09 & $\mathrm{pCi} / \mathrm{L}$ \\
\hline & Technetium & 23.00 & 62.00 & 35.50 & 17.97 & $\mathrm{pCi} / \mathrm{L}$ \\
\hline & Uranium & $1.00 \mathrm{U}$ & 8.90 & & & $\mu \mathrm{g} / \mathrm{L}$ \\
\hline \multirow[t]{4}{*}{$X 701-25 G$} & Gross Alpha & $7.00 \mathrm{U}$ & 8.00 & & & $\mathrm{pCi} / \mathrm{L}$ \\
\hline & Gross Beta & $8.00 \mathrm{U}$ & $11.00 \mathrm{U}$ & & & $\mathrm{pCi} / \mathrm{L}$ \\
\hline & Technetium & $22.00 \mathrm{U}$ & $22.00 \mathrm{U}$ & & & $\mathrm{pCi} / \mathrm{L}$ \\
\hline & Uranium & $1.00 \mathrm{U}$ & $1.00 \mathrm{U}$ & & & $\mu \mathrm{g} / \mathrm{L}$ \\
\hline \multirow[t]{4}{*}{$X 701-30 G$} & Gross Alpha & $6: 00 \mathrm{U}$ & 8.00 & & & $\mathrm{pCi} / \mathrm{L}$ \\
\hline & Gross Beta & $11.00 \mathrm{U}$ & 20.00 & 13.25 & 4.50 & $\mathrm{pCi} / \mathrm{L}$ \\
\hline & Technetium & $22.00 \mathrm{U}$ & $22.00 \mathrm{U}$ & & & $\mathrm{pCi} / \mathrm{L}$ \\
\hline & Uranium & $1.00 \mathrm{U}$ & $1.00 \mathrm{U}$ & & & $\mu \mathrm{g} / \mathrm{L}$ \\
\hline \multirow[t]{4}{*}{$X 701-31 G$} & Gross Alpha & $4.00 \mathrm{U}$ & $5.00 \mathrm{U}$ & & & $\mathrm{pCi} / \mathrm{L}$ \\
\hline & Gross Beta & $8.00 \mathrm{U}$ & $11.00 \mathrm{U}$ & & & $\mathrm{pCi} / \mathrm{L}$ \\
\hline & Technetium & $22.00 \mathrm{U}$ & $22.00 \mathrm{U}$ & & & $\mathrm{pCi} / \mathrm{L}$ \\
\hline & Uranium & $1.00 \mathrm{U}$ & $1.00 \mathrm{U}$ & & & $\mu \mathrm{g} / \mathrm{L}$ \\
\hline \multirow[t]{2}{*}{$X 701-32 G$} & Gross Alpha & $4.00 \mathrm{U}$ & $5.00 \mathrm{U}$ & & & $\mathrm{pCi} / \mathrm{L}$ \\
\hline & Gross Beta & $8.00 \mathrm{U}$ & $11.00 \mathrm{U}$ & & & $\mathrm{pCi} / \mathrm{L}$ \\
\hline
\end{tabular}


Table 3.2. DOE/PORTS groundwater monitoring for 1995: results for radionuclide parameters at $X-701 B$ holding pond (continued)

\begin{tabular}{|c|c|c|c|c|c|c|}
\hline Well & Parameter & Min & Max & Mean & $\begin{array}{l}\text { Standard } \\
\text { Deviation }\end{array}$ & Units \\
\hline \multicolumn{7}{|c|}{ Gallia Wells (cont) } \\
\hline \multirow[t]{2}{*}{$\mathrm{X} 701-32 \mathrm{G}$} & Technetium & $22.00 \mathrm{U}$ & $22.00 \mathrm{U}$ & & & $\mathrm{pCi} / \mathrm{L}$ \\
\hline & Uranium & $1.00 \mathrm{U}$ & $1.00 \mathrm{U}$ & & & $\mu \mathrm{g} / \mathrm{L}$ \\
\hline \multirow[t]{4}{*}{$X 701-38 G$} & Gross Alpha & $5.70 \mathrm{U}$ & $7.00 \mathrm{U}$ & & & pCilL \\
\hline & Gross Beta & $8.00 \mathrm{U}$ & $11.00 \mathrm{U}$ & & & pCilL \\
\hline & Technetium & $22.00 \mathrm{U}$ & $22.00 \mathrm{U}$ & & & $\mathrm{pCi} / \mathrm{L}$ \\
\hline & Uranium & $1.00 \mathrm{U}$ & $1.00 \mathrm{U}$ & & & $\mu \mathrm{g} / \mathrm{L}$ \\
\hline \multirow[t]{4}{*}{$\times 701-48 G$} & Gross Alpha & $4.00 \mathrm{U}$ & 8.00 & & & $\mathrm{pCi} / \mathrm{L}$ \\
\hline & Gross Beta & $8.00 \mathrm{U}$ & $11.00 \mathrm{U}$ & & & $\mathrm{pCi} / \mathrm{L}$ \\
\hline & Technetium & $22.00 \mathrm{U}$ & $22.00 \mathrm{U}$ & & & $\mathrm{pCi} / \mathrm{L}$ \\
\hline & Uranium & $1.00 \mathrm{U}$ & $1.00 \mathrm{U}$ & & & $\mu \mathrm{g} / \mathrm{L}$ \\
\hline \multirow[t]{4}{*}{$\mathrm{X} 701-49 \mathrm{G}$} & Gross Alpha & $5.00 \mathrm{U}$ & 11.00 & 8.67 & 3.21 & $\mathrm{pCi} / \mathrm{L}$ \\
\hline & Gross Beta & $8.00 \mathrm{U}$ & 10.00 & 8.67 & 1.15 & $\mathrm{pCi} / \mathrm{L}$ \\
\hline & Technetium & $22.00 \mathrm{U}$ & $22.00 \mathrm{U}$ & & & $\mathrm{pCi} / \mathrm{L}$ \\
\hline & Uranium & 1.60 & 3.00 & 2.20 & 0.72 & $\mu \mathrm{g} / \mathrm{L}$ \\
\hline \multirow[t]{4}{*}{$\mathrm{X} 701-\mathrm{BW} 1 \mathrm{G}$} & Gross Alpha & $6.00 \mathrm{U}$ & $6.00 \mathrm{U}$ & & & $\mathrm{pCi} / \mathrm{L}$ \\
\hline & Gross Beta & $8.00 \mathrm{U}$ & $8.00 \mathrm{U}$ & & & $\mathrm{pCi} / \mathrm{L}$ \\
\hline & Technetium & $22.00 \mathrm{U}$ & $22.00 \mathrm{U}$ & & & $\mathrm{pCi} / \mathrm{L}$ \\
\hline & Uranium & $1.00 \mathrm{U}$ & $1.00 \mathrm{U}$ & & & $\mu \mathrm{g} / \mathrm{L}$ \\
\hline \multirow[t]{4}{*}{ X701-BW4G } & Gross Alpha & $9.00 \mathrm{U}$ & $9.00 \mathrm{U}$ & & & $\mathrm{pCi} / \mathrm{L}$ \\
\hline & Gross Beta & 27.00 & 55.00 & 36.75 & 12.45 & $\mathrm{pCi} / \mathrm{L}$ \\
\hline & Technetium & 28.00 & 53.00 & 42.25 & 12.28 & $\mathrm{pCi} / \mathrm{L}$ \\
\hline & Uranium & $1.00 \mathrm{U}$ & $1.00 \mathrm{U}$ & & & $\mu \mathrm{g} / \mathrm{L}$ \\
\hline
\end{tabular}

Data Qualifer (U)--Parameter not detected. Result is attainable detection limit for the sample. Mean and standard deviation functions not calculated if at least $50 \%$ of the results for the parameter were below detection. 
Table 3.3. DOE/PORTS groundwater monitoring for 1995: results for volatile organic compounds at $X-749$

\begin{tabular}{|c|c|c|c|c|c|c|}
\hline Well & Parameter & Min & Max & Mean & $\begin{array}{l}\text { Standard } \\
\text { Deviation }\end{array}$ & Units \\
\hline \multicolumn{7}{|c|}{ Berea Wells } \\
\hline \multirow[b]{8}{*}{$\mathrm{X} 749-50 \mathrm{~B}$} & 1,1,1-Trichloroethane & $2.00 \mathrm{U}$ & $2.00 \mathrm{U}$ & & & $\mu \mathrm{g} / \mathrm{L}$ \\
\hline & 1,1-Dichloroethane & $2.00 \mathrm{U}$ & $2.00 \mathrm{U}$ & & & $\mu \mathrm{g} / \mathrm{L}$ \\
\hline & 1,1-Dichloroethene & $2.00 \mathrm{U}$ & $2.00 \mathrm{U}$ & & & $\mu \mathrm{g} / \mathrm{L}$ \\
\hline & 1,2-Dichloroethane & $2.00 \mathrm{U}$ & $2.00 \mathrm{U}$ & & & $\mu \mathrm{g} / \mathrm{L}$ \\
\hline & Chloroform & $2.00 \mathrm{U}$ & $2.00 \mathrm{U}$ & & & $\mu \mathrm{g} / \mathrm{L}$ \\
\hline & Freon 113 & $2.00 \mathrm{U}$ & $2.00 \mathrm{U}$ & & & $\mu \mathrm{g} / \mathrm{L}$ \\
\hline & Trichloroethene & $2.00 \mathrm{U}$ & $2.00 \mathrm{U}$ & & & $\mu \mathrm{g} / \mathrm{L}$ \\
\hline & 1,1,1-Trichloroethane & $2.00 \mathrm{U}$ & $2.00 \mathrm{U}$ & & & $\mu \mathrm{g} / \mathrm{L}$ \\
\hline \multirow[b]{8}{*}{$X 749-51 B$} & 1,1-Dichloroethane & 3.00 & 7.00 & 4.50 & 1.73 & $\mu \mathrm{g} / \mathrm{L}$ \\
\hline & 1,1-Dichloroethene & $2.00 \mathrm{U}$ & $2.00 \mathrm{U}$ & & & $\mu \mathrm{g} / \mathrm{L}$ \\
\hline & 1,2-Dichloroethane & $2.00 \mathrm{U}$ & 4.00 & 2.50 & 1.00 & $\mu \mathrm{g} / \mathrm{L}$ \\
\hline & 1,2-Dichloroethenes (cis \& t) & $2.00 \mathrm{U}$ & $2.00 \mathrm{U}$ & & & $\mu \mathrm{g} / \mathrm{L}$ \\
\hline & Chloroform & $2.00 \mathrm{U}$ & $2.00 \mathrm{U}$ & & & $\mu \mathrm{g} / \mathrm{L}$ \\
\hline & Freon 113 & $2.00 \mathrm{U}$ & $2.00 \mathrm{U}$ & & & $\mu \mathrm{g} / \mathrm{L}$ \\
\hline & Trichloroethene & $2.00 \mathrm{U}$ & $2.00 \mathrm{U}$ & & & $\mu \mathrm{g} / \mathrm{L}$ \\
\hline & 1,1,1-Trichloroethane & $2.00 \mathrm{U}$ & $2.00 \mathrm{U}$ & & & $\mu \mathrm{g} / \mathrm{L}$ \\
\hline \multirow{13}{*}{$X 749-54 B$} & 1,1-Dichloroethane & $2.00 \mathrm{U}$ & $2.00 \mathrm{U}$ & & & $\mu \mathrm{g} / \mathrm{L}$ \\
\hline & 1,1-Dichloroethene & $2.00 \mathrm{U}$ & $2.00 \mathrm{U}$ & & & $\mu \mathrm{g} / \mathrm{L}$ \\
\hline & 1,2-Dichloroethane & $2.00 \mathrm{U}$ & $2.00 \mathrm{U}$ & & & $\mu \mathrm{g} / \mathrm{L}$ \\
\hline & 1,2-Dichloroethenes (cis \& $t$ ) & $2.00 \mathrm{U}$ & $2.00 \mathrm{U}$ & & & $\mu \mathrm{g} / \mathrm{L}$ \\
\hline & Chloroform & $2.00 \mathrm{U}$ & $2.00 \mathrm{U}$ & & & $\mu \mathrm{g} / \mathrm{L}$ \\
\hline & Freon 113 & $2.00 \mathrm{U}$ & $2.00 \mathrm{U}$ & & & $\mu \mathrm{g} / \mathrm{L}$ \\
\hline & Trichloroethene & $2.00 \mathrm{U}$ & $2.00 \mathrm{U}$ & & & $\mu \mathrm{g} / \mathrm{L}$ \\
\hline & 1,1,1-Trichloroethane & $2.00 \mathrm{U}$ & $2.00 \mathrm{U}$ & & & $\mu \mathrm{g} / \mathrm{L}$ \\
\hline & 1,1-Dichloroethane & $2.00 \mathrm{U}$ & $2.00 \mathrm{U}$ & & & $\mu \mathrm{g} / \mathrm{L}$ \\
\hline & 1,1-Dichloroethene & $2.00 \mathrm{U}$ & $2.00 \mathrm{U}$ & & & $\mu \mathrm{g} / \mathrm{L}$ \\
\hline & 1,2-Dichloroethane & $2.00 \mathrm{U}$ & $2.00 \mathrm{U}$ & & & $\mu \mathrm{g} / \mathrm{L}$ \\
\hline & 1,2-Dichloroethenes (cis \& t) & $2.00 \mathrm{U}$ & $2.00 \mathrm{U}$ & & & $\mu \mathrm{g} / \mathrm{L}$ \\
\hline & Chloroform & $2.00 \mathrm{U}$ & $2.00 \mathrm{U}$ & & & $\mu \mathrm{g} / \mathrm{L}$ \\
\hline
\end{tabular}


Table 3.3. DOE/PORTS groundwater monitoring for 1995: results for volatile organic compounds at X-749 (continued)

\begin{tabular}{|c|c|c|c|c|c|c|}
\hline Well & Parameter & Min & $\operatorname{Max}$ & Mean & $\begin{array}{l}\text { Standard } \\
\text { Deviation }\end{array}$ & Units \\
\hline \multicolumn{7}{|c|}{ Berea Wells (cont) } \\
\hline \multirow[t]{2}{*}{ X749-54B } & Freon 113 & $2.00 \mathrm{U}$ & $2.00 \mathrm{U}$ & & & $\mu \mathrm{g} / \mathrm{L}$. \\
\hline & Trichloroethene & $2.00 \mathrm{U}$ & $2.00 \mathrm{U}$ & & & $\mu \mathrm{g} / \mathrm{L}$ \\
\hline \multirow[t]{8}{*}{$X 749-60 B$} & 1,1,1-Trichloroethane & $2.00 \mathrm{U}$ & $2.00 \mathrm{U}$ & & & $\mu \mathrm{g} / \mathrm{L}$ \\
\hline & 1,1-Dichloroethane & $2.00 \mathrm{U}$ & $2.00 \mathrm{U}$ & & & $\mu \mathrm{g} / \mathrm{L}$ \\
\hline & 1,1-Dichloroethene & $2.00 \mathrm{U}$ & $2.00 \mathrm{U}$ & & & $\mu g / L$ \\
\hline & 1,2-Dichloroethane & $2.00 \mathrm{U}$ & $2.00 \mathrm{U}$ & & & $\mu \mathrm{g} / \mathrm{L}$ \\
\hline & 1,2-Dichloroethenes (cis \& $t$ ) & $2.00 \mathrm{U}$ & $2,00 \mathrm{U}$ & & & $\mu \mathrm{g} / \mathrm{L}$ \\
\hline & Chloroform & $2.00 \mathrm{U}$ & $2.00 \mathrm{U}$ & & & $\mu \mathrm{g} / \mathrm{L}$ \\
\hline & Freon 113 & $2.00 \mathrm{U}$ & $2.00 \mathrm{U}$ & & & $\mu \mathrm{g} / \mathrm{L}$ \\
\hline & Trichloroethene & $2.00 \mathrm{U}$ & $2.00 \mathrm{U}^{\prime}$ & & & $\mu \mathrm{g} / \mathrm{L}$ \\
\hline \multirow[t]{9}{*}{ X749-64B } & 1,1,1-Trichloroethane & $2.00 \mathrm{U}$ & $2.00 \mathrm{U}$ & & & $\mu \mathrm{g} / \mathrm{L}$ \\
\hline & 1,1-Dichloroethane & $2.00 \mathrm{U}$ & $2.00 \mathrm{U}$ & & & $\mu \mathrm{g} / \mathrm{L}$ \\
\hline & 1,1-Dichloroethene & $2.00 \mathrm{U}$ & $2.00 \mathrm{U}$ & & & $\mu g / L$ \\
\hline & 1,2-Dichloroethane & $2.00 \mathrm{U}$ & $2.00 \mathrm{U}$ & & & $\mu g / L$ \\
\hline & 1,2-Dichloroethenes (cis \& t) & $2.00 \mathrm{U}$ & $2.00 \mathrm{U}$ & & & $\mu \mathrm{g} / \mathrm{L}$ \\
\hline & Chloroform & $2.00 \mathrm{U}$ & $2.00 \mathrm{U}$ & & & $\mu \mathrm{g} / \mathrm{L}$ \\
\hline & Freon 113 & $2.00 \mathrm{U}$ & $2.00 \mathrm{U}$ & & & $\mu \mathrm{g} / \mathrm{L}$ \\
\hline & Trichloroethene & $2.00 \mathrm{U}$ & $2.00 \mathrm{U}$ & & & $\mu g / L$ \\
\hline & & Gallia We & & & & \\
\hline \multirow[t]{7}{*}{$\mathrm{X} 120-08 \mathrm{G}$} & 1,1,1-Trichloroethane & $10.00 \mathrm{U}$ & 55.00 & 42.25 & 21.55 & $\mu \mathrm{g} / \mathrm{L}$ \\
\hline & 1,1-Dichloroethane & 15.00 & 18.00 & 16.50 & 1.73 & $\mu \mathrm{g} / \mathrm{L}$ \\
\hline & 1,1-Dichloroethene & 72.00 & 78.00 & 75.00 & 3.46 & $\mu \mathrm{g} / \mathrm{L}$ \\
\hline & 1,2-Dichloroethane & 3.00 & $10.00 \mathrm{U}$ & & & $\mu \mathrm{g} / \mathrm{L}$ \\
\hline & Chloroform & 2.00 & $10.00 \mathrm{U}$ & & & $\mu \mathrm{g} / \mathrm{L}$ \\
\hline & Freon 113 & 5.00 & $10.00 \mathrm{U}$ & & & $\mu \mathrm{g} / \mathrm{L}$ \\
\hline & Trichloroethene & 12.00 & 12.00 & 12.00 & 0.00 & $\mu \mathrm{g} / \mathrm{L}$ \\
\hline $\mathrm{X} 749-04 \mathrm{G}$ & 1,1,1-Trichloroethane & $20.00 \mathrm{U}$ & $20.00 \mathrm{U}$ & & & $\mu \mathrm{g} / \mathrm{L}$ \\
\hline
\end{tabular}


Table 3.3. DOE/PORTS groundwater monitoring for 1995: results for volatile organic compounds at X-749 (continued)

\begin{tabular}{|c|c|c|c|c|c|c|}
\hline Well & Parameter & Min & Max & Mean & $\begin{array}{l}\text { Standard } \\
\text { Deviation }\end{array}$ & Units \\
\hline \multicolumn{7}{|c|}{ Gallia Wells (cont) } \\
\hline \multirow[t]{7}{*}{$\mathrm{X} 749-04 \mathrm{G}$} & 1,1-Dichloroethane & $20.00 \mathrm{U}$ & $20.00 \mathrm{U}$ & & & $\mu \mathrm{g} / \mathrm{L}$ \\
\hline & 1,1-Dichloroethene & $20.00 \mathrm{U}$ & $20.00 \mathrm{U}$ & & & $\mu \mathrm{g} / \mathrm{L}$ \\
\hline & 1,2-Dichloroethane & $20.00 \mathrm{U}$ & $20.00 \mathrm{U}$ & & & $\mu \mathrm{g} / \mathrm{L}$ \\
\hline & 1,2-Dichloroethenes (cis \& t) & $20.00 \mathrm{U}$ & $20.00 \mathrm{U}$ & & & $\mu \mathrm{g} / \mathrm{L}$ \\
\hline & Chloroform & $20.00 \mathrm{U}$ & $20.00 \mathrm{U}$ & & & $\mu \mathrm{g} / \mathrm{L}$ \\
\hline & Freon 113 & $20.00 \mathrm{U}$ & $20.00 \mathrm{U}$ & & & $\mu \mathrm{g} / \mathrm{L}$ \\
\hline & Trichloroethene & 210.00 & 430.00 & 290.00 & 121.66 & $\mu \mathrm{g} / \mathrm{L}$ \\
\hline \multirow[t]{8}{*}{ X749-06G } & 1,1,1-Trichloroethane & 3200.00 & 3900.00 & 3500.00 & 355.90 & $\mu g / L$ \\
\hline & 1,1-Dichloroethane & 1500.00 & 1800.00 & 1625.00 & 150.00 & $\mu \mathrm{g} / \mathrm{L}$ \\
\hline & 1,1-Dichloroethene & 1600.00 & 2100.00 & 1750.00 & 238.05 & $\mu \mathrm{g} / \mathrm{L}$ \\
\hline & 1,2-Dichloroethane & $200.00 \mathrm{U}$ & $400.00 \mathrm{U}$ & & & $\mu g / L$ \\
\hline & 1,2-Dichloroethenes (cis \& $t$ & 300.00 & 300.00 & 300.00 & & $\mu \mathrm{g} / \mathrm{L}$ \\
\hline & Chloroform & $200.00 \mathrm{U}$ & $400.00 \mathrm{U}$ & & & $\mu \mathrm{g} / \mathrm{L}$ \\
\hline & Freon 113 & 480.00 & 600.00 & 530.00 & 60.00 & $\mu \mathrm{g} / \mathrm{L}$ \\
\hline & Trichloroethene & 4700.00 & 5800.00 & 5100.00 & 522.81 & $\mu \mathrm{g} / \mathrm{L}$ \\
\hline \multirow[t]{8}{*}{ X749-07G } & 1,1,1-Trichloroethane & 1200.00 & 1200.00 & 1200.00 & 0.00 & $\mu \mathrm{g} / \mathrm{L}$ \\
\hline & 1,1-Dichloroethane & 610.00 & 1600.00 & 990.00 & 533.57 & $\mu \mathrm{g} / \mathrm{L}$ \\
\hline & 1,1-Dichloroethene & 600.00 & 680.00 & 640.00 & 40.00 & $\mu \mathrm{g} / \mathrm{L}$ \\
\hline & 1,2-Dichloroethane & 260.00 & 620.00 & 393.33 & 197.32 & $\mu \mathrm{g} / \mathrm{L}$ \\
\hline & 1,2-Dichloroethenes (cis \& t) & $200.00 \mathrm{U}$ & $200.00 \mathrm{U}$ & & & $\mu \mathrm{g} / \mathrm{L}$ \\
\hline & Chloroform & $100.00 \mathrm{U}$ & $200.00 \mathrm{U}$ & & & $\mu \mathrm{g} / \mathrm{L}$ \\
\hline & Freon 113 & $200.00 \mathrm{U}$ & 240.00 & 216.67 & 20.82 & $\mu \mathrm{g} / \mathrm{L}$ \\
\hline & Trichloroethene & 1300.00 & 1800.00 & 1533.33 & 251.66 & $\mu \mathrm{g} / \mathrm{L}$ \\
\hline \multirow[t]{5}{*}{ X749-08G } & 1,1,1-Trichloroethane & 700.00 & 820.00 & 740.00 & 48.99 & $\mu \mathrm{g} / \mathrm{L}$ \\
\hline & 1;1-Dichloroethane & 25.00 & $100.00 U$ & 56.00 & 40.22 & $\mu \mathrm{g} / \mathrm{L}$ \\
\hline & 1,1-Dichloroethene & $100.00 \mathrm{U}$ & 540.00 & 420.00 & 181.52 & $\mu \mathrm{g} / \mathrm{L}$ \\
\hline & 1,2-Dichloroethane & $20.00 \mathrm{U}$ & $100.00 \mathrm{U}$ & & & $\mu \mathrm{g} / \mathrm{L}$ \\
\hline & 1,2-Dichloroethenes (cis \& t) & 51.00 & 51.00 & 51.00 & & $\mu \mathrm{g} / \mathrm{L}$ \\
\hline
\end{tabular}


Table 3.3. DOE/PORTS groundwater monitoring for 1995: results for volatile organic compounds at $X-749$ (continued)

\begin{tabular}{|c|c|c|c|c|c|c|}
\hline Well & Parameter & Min & Max & Mean & $\begin{array}{l}\text { Standard } \\
\text { Deviation }\end{array}$ & Units \\
\hline \multicolumn{7}{|c|}{ Gallia Wells (cont) } \\
\hline \multirow{3}{*}{$\mathrm{X} 749-08 \mathrm{G}$} & Chloroform & $20.00 \mathrm{U}$ & $100.00 \mathrm{U}$ & & & $\mu \mathrm{g} / \mathrm{L}$ \\
\hline & Freon 113 & 100.00 & 110.00 & 106.00 & 5.48 & $\mu \mathrm{g} / \mathrm{L}$ \\
\hline & Trichloroethene & 460.00 & 530.00 & 494.00 & 32.09 & $\mu \mathrm{g} / \mathrm{L}$ \\
\hline \multirow[t]{8}{*}{$\times 749-09 \mathrm{G}$} & 1,1,1-Trichloroethane & $2.00 \mathrm{U}$ & $2.00 \mathrm{U}$ & & & $\mu \mathrm{g} / \mathrm{L}$ \\
\hline & 1,1-Dichloroethane & 22.00 & 27.00 & 24.25 & 2.06 & $\mu \mathrm{g} / \mathrm{L}$ \\
\hline & 1,1-Dichloroethene & 8.00 & 11.00 & 8.75 & 1.50 & $\mu \mathrm{g} / \mathrm{L}$ \\
\hline & 1,2-Dichloroethane & $2.00 \mathrm{U}$ & $2.00 \mathrm{U}$ & & & $\mu g / L$ \\
\hline & 1,2-Dichloroethenes (cis \& $\mathrm{t}$ & 4.00 & 4.00 & 4.00 & & $\mu \mathrm{g} / \mathrm{L}$ \\
\hline & Chloroform & $2.00 \mathrm{U}$ & $2.00 \mathrm{U}$ & & & $\mu \mathrm{g} / \mathrm{L}$ \\
\hline & Freon 113 & $2.00 \mathrm{U}$ & $2.00 \mathrm{U}$ & & & $\mu \mathrm{g} / \mathrm{L}$ \\
\hline & Trichloroethene & $2.00 \mathrm{U}$ & $2.00 \mathrm{U}$ & & & $\mu \mathrm{g} / \mathrm{L}$ \\
\hline \multirow[t]{8}{*}{ X749-10G } & 1,1,1-Trichloroethane & 33.00 & 270.00 & 161.00 & 119.64 & $\mu \mathrm{g} / \mathrm{L}$ \\
\hline & 1,1-Dichloroethane & 9.00 & $200.00 \mathrm{U}$ & 78.33 & 105.71 & $\mu \mathrm{g} / \mathrm{L}$ \\
\hline & 1,1-Dichloroethene & 9.00 & $200.00 \mathrm{U}$ & 96.67 & 96.46 & $\mu \mathrm{g} / \mathrm{L}$ \\
\hline & 1,2-Dichloroethane & $2.00 \mathrm{U}$ & $200.00 \mathrm{U}$ & & & $\mu \mathrm{g} / \mathrm{L}$ \\
\hline & 1,2-Dichloroethenes (cis \& $t$ ) & 10.00 & 10.00 & 10.00 & & $\mu \mathrm{g} / \mathrm{L}$ \\
\hline & Chloroform & $2.00 \mathrm{U}$ & $200.00 \mathrm{U}$ & & & $\mu \mathrm{g} / \mathrm{L}$ \\
\hline & Freon 113 & 4.00 & $200.00 \mathrm{U}$ & & & $\mu \mathrm{g} / \mathrm{L}$ \\
\hline & Trichloroethene & 81.00 & 340.00 & 230.33 & 133.98 & $\mu \mathrm{g} / \mathrm{L}$ \\
\hline \multirow[t]{8}{*}{$X 749-13 G$} & 1,1,1-Trichloroethane & 120.00 & 140.00 & 130.00 & 10.00 & $\mu \mathrm{g} / \mathrm{L}$ \\
\hline & 1,1-Dichloroethane & 6.00 & 100.00 & 42.00 & 50.71 & $\mu \mathrm{g} / \mathrm{L}$ \\
\hline & 1,1-Dichloroethene & $20.00 \mathrm{U}$ & 120.00 & 80.00 & 52.92 & $\mu \mathrm{g} / \mathrm{L}$ \\
\hline & 1,2-Dichloroethane & $4.00 \mathrm{U}$ & $20.00 \mathrm{U}$ & & & $\mu \mathrm{g} / \mathrm{L}$ \\
\hline & 1,2-Dichloroethenes (cis \& $t$ ) & $20.00 \mathrm{U}$ & $20.00 \mathrm{U}$ & & & $\mu \mathrm{g} / \mathrm{L}$ \\
\hline & Chloroform & 7.00 & $20.00 \mathrm{U}$ & & & $\mu \mathrm{g} / \mathrm{L}$ \\
\hline & Freon 113 & 35.00 & 46.00 & 41.33 & 5.69 & $\mu \mathrm{g} / \mathrm{L}$ \\
\hline & Trichloroethene & 170.00 & 200.00 & 183.33 & 15.28 & $\mu \mathrm{g} / \mathrm{L}$ \\
\hline $\mathrm{X} 749-21 \mathrm{G}$ & 1,1,1-Trichloroethane & $2.00 \mathrm{U}$ & $2.00 \mathrm{U}$ & & & $\mu g / L$ \\
\hline
\end{tabular}


Table 3.3. DOE/PORTS groundwater monitoring for 1995: results for volatile organic compounds at X-749 (continued)

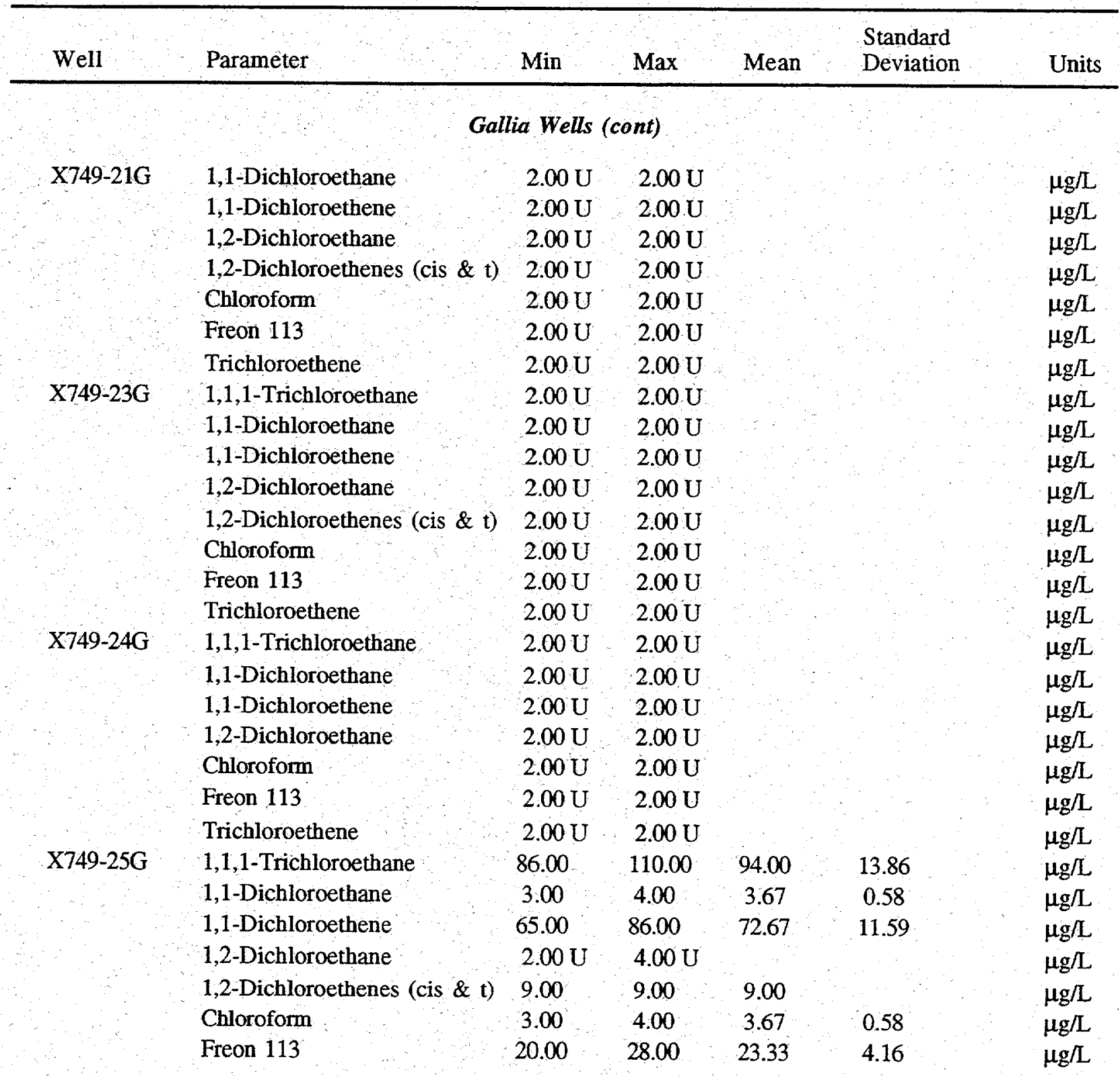


Table 3.3. DOE/PORTS groundwater monitoring for 1995: results for volatile organic compounds at X-749 (continued)

\begin{tabular}{|c|c|c|c|c|c|c|}
\hline Well & Parameter & Min & $\operatorname{Max}$ & Mean & $\begin{array}{l}\text { Standard } \\
\text { Deviation }\end{array}$ & Units \\
\hline \multicolumn{7}{|c|}{ Gallia Wells (cont) } \\
\hline $\mathrm{X} 749-25 \mathrm{G}$ & Trichloroethene & 94.00 & 120.00 & 104.33 & 13.80 & $\mu \mathrm{g} / \mathrm{L}$ \\
\hline \multirow[t]{7}{*}{$X 749-26 G$} & 1,1,1-Trichloroethane & 130.00 & $200.00 \mathrm{U}$ & 147.50 & 35.00 & $\mu \mathrm{g} / \mathrm{L}$ \\
\hline & 1,1-Dichloroethane & 220.00 & 300.00 & 240.00 & 40.00 & $\mu \mathrm{g} / \mathrm{L}$ \\
\hline & 1,1-Dichloroethene & 94.00 & $200.00 \mathrm{U}$ & 120.50 & 53.00 & $\mu \mathrm{g} / \mathrm{L}$ \\
\hline & 1,2-Dichloroethane & 93.00 & $200.00 \mathrm{U}$ & 119.75 & 53.50 & $\mu \mathrm{g} / \mathrm{L}$ \\
\hline & Chloroform & $20.00 \mathrm{U}$ & $200.00 \mathrm{U}$ & & & $\mu \mathrm{g} / \mathrm{L}$ \\
\hline & Freon 113 & 48.00 & $200.00 \mathrm{U}$ & 86.00 & 76.00 & $\mu \mathrm{g} / \mathrm{L}$ \\
\hline & Trichloroethene & 320.00 & 390.00 & 337.50 & 35.00 & $\mu \mathrm{g} / \mathrm{L}$ \\
\hline \multirow[t]{7}{*}{$\times 749-32 \mathrm{G}$} & 1,1,1-Trichloroethane & 1100.00 & 1400.00 & 1200.00 & 173.21 & $\mu \mathrm{g} / \mathrm{L}$ \\
\hline & 1;1-Dichloroethane & 320.00 & 390,00 & 343.33 & 40.41 & $\mu \mathrm{g} / \mathrm{L}$ \\
\hline & 1,1-Dichloroethene & 780.00 & 890.00 & 816.67 & 63.51 & $\mu \mathrm{g} / \mathrm{L}$ \\
\hline & 1,2-Dichloroethane & $100.00 \mathrm{U}$ & $200.00 \mathrm{U}$ & & & $\mu \mathrm{g} / \mathrm{L}$ \\
\hline & Chloroform & $100.00 \mathrm{U}$ & $200.00 \mathrm{U}$ & & & $\mu \mathrm{g} / \mathrm{L}$ \\
\hline & Freon 113 & 290.00 & 340.00 & 306.67 & 28.87 & $\mu \mathrm{g} / \mathrm{L}$ \\
\hline & Trichloroethene & 1400.00 & 1600.00 & 1466.67 & 115.47 & $\mu \mathrm{g} / \mathrm{L}$ \\
\hline \multirow[t]{7}{*}{$\mathrm{X} 749-36 \mathrm{G}$} & 1,1,1-Trichloroethane & 580.00 & 660.00 & 633.33 & 46.19 & $\mu \mathrm{g} / \mathrm{L}$ \\
\hline & 1,1-Dichloroethane & 130.00 & 140.00 & 136.67 & 5.77 & $\mu \mathrm{g} / \mathrm{L}$ \\
\hline & 1,1-Dichloroethene & 470.00 & $490: 00$ & 483.33 & 11.55 & $\mu \mathrm{g} / \mathrm{L}$ \\
\hline & 1,2-Dichloroethane & $20.00 \mathrm{U}$ & $20.00 \mathrm{U}$ & & & $\mu \mathrm{g} / \mathrm{L}$ \\
\hline & Chloroform & $20.00 \mathrm{U}$ & $20.00 \mathrm{U}$ & & & $\mu \mathrm{g} / \mathrm{L}$ \\
\hline & Freon 113 & 94.00 & 110.00 & 104.67 & 9.24 & $\mu \mathrm{g} / \mathrm{L}$ \\
\hline & Trichloroethene & 280.00 & 360.00 & 333.33 & 46.19 & $\mu \mathrm{g} / \mathrm{L}$ \\
\hline \multirow[t]{6}{*}{$\mathrm{X} 749-37 \mathrm{G}$} & 1,1,1-Trichloroethane & 420.00 & 510.00 & 453.33 & 49.33 & $\mu \mathrm{g} / \mathrm{L}$ \\
\hline & 1,1-Dichloroethane & 110.00 & 130.00 & 116.67 & 11.55 & $\mu \mathrm{g} / \mathrm{L}$ \\
\hline & 1,1-Dichloroethene & 360.00 & 420.00 & 383.33 & 32.15 & $\mu \mathrm{g} / \mathrm{L}$ \\
\hline & 1,2-Dichloroethane & $20.00 \mathrm{U}$ & $20.00 \mathrm{U}$ & & & $\mu \mathrm{g} / \mathrm{L}$ \\
\hline & 1,2-Dichloroethenes (cis \& $\mathrm{t}$ ) & $20.00 \mathrm{U}$ & $20.00 \mathrm{U}$ & & & $\mu \mathrm{g} / \mathrm{L}$ \\
\hline & Chloroform & $20.00 \mathrm{U}$ & $20.00 \mathrm{U}$ & & & $\mu \mathrm{g} / \mathrm{L}$ \\
\hline
\end{tabular}


Table 3.3. DOE/PORTS groundwater monitoring for 1995: results for volatile organic compounds at X-749 (continued)

\begin{tabular}{|c|c|c|c|c|c|c|}
\hline Well & Parameter & Min & $\operatorname{Max}$ & Mean & $\begin{array}{l}\text { Standard } \\
\text { Deviation }\end{array}$ & Units \\
\hline \multicolumn{7}{|c|}{ Gallia Wells (cont) } \\
\hline \multirow{2}{*}{$\mathrm{X} 749-37 \mathrm{G}$} & Freon 113 & 68.00 & 84.00 & 74.67 & 8.33 & $\mu \mathrm{g} / \mathrm{L}$ \\
\hline & Trichloroethene & 320.00 & 390.00 & 346.67 & 37.86 & $\mu \mathrm{g} / \mathrm{L}$ \\
\hline \multirow[t]{8}{*}{$X 749-43 G$} & 1,1,1-Trichloroethane & $2.00 \mathrm{U}$ & $2.00 \mathrm{U}$ & & & $\mu \mathrm{g} / \mathrm{L}$ \\
\hline & 1,1-Dichloroethane & $2.00 \mathrm{U}$ & $2.00 \mathrm{U}$ & & & $\mu \mathrm{g} / \mathrm{L}$ \\
\hline & 1,1-Dichloroethene & $2.00 \mathrm{U}$ & $2.00 \mathrm{U}$ & & & $\mu \mathrm{g} / \mathrm{L}$ \\
\hline & 1,2-Dichloroethane & $2.00 \mathrm{U}$ & $2.00 \mathrm{U}$ & & . & $\mu \mathrm{g} / \mathrm{L}$ \\
\hline & 1,2-Dichloroethenes (cis \& $t$ ) & $2.00 \mathrm{U}$ & $2.00 \mathrm{U}$ & & & $\mu \mathrm{g} / \mathrm{L}$ \\
\hline & Chloroform & $2.00 \mathrm{U}$ & $2.00 \mathrm{U}$ & & & $\mu \mathrm{g} / \mathrm{L}$ \\
\hline & Freon 113 & $2.00 \mathrm{U}$ & $2.00 \mathrm{U}$ & & & $\mu \mathrm{g} / \mathrm{L}$ \\
\hline & Trichloroethene & $2.00 \mathrm{U}$ & $2.00 \mathrm{U}$ & & & $\mu \mathrm{g} / \mathrm{L}$ \\
\hline \multirow[t]{8}{*}{$X 749-44 G$} & 1,1,1-Trichloroethane & $2.00 \mathrm{U}$ & $2.00 \mathrm{U}$ & & & $\mu \mathrm{g} / \mathrm{L}$ \\
\hline & 1,1-Dichloroethane & $2.00 \mathrm{U}$ & $2.00 \mathrm{U}$ & & & $\mu \mathrm{g} / \mathrm{L}$ \\
\hline & 1,1-Dichloroethene & $2.00 \mathrm{U}$ & $2.00 \mathrm{U}$ & & & $\mu \mathrm{g} / \mathrm{L}$ \\
\hline & 1,2-Dichloroethane & $2.00 \mathrm{U}$ & $2.00 \mathrm{U}$ & & & $\mu \mathrm{g} / \mathrm{L}$ \\
\hline & 1,2-Dichloroethenes (cis \& t) & $2.00 \mathrm{U}$ & $2.00 \mathrm{U}$ & & & $\mu \mathrm{g} / \mathrm{L}$ \\
\hline & Chloroform & $2.00 \mathrm{U}$ & $2.00 \mathrm{U}$ & & & $\mu \mathrm{g} / \mathrm{L}$ \\
\hline & Freon 113 & $2.00 \mathrm{U}$ & $2.00 \mathrm{U}$ & & & $\mu \mathrm{g} / \mathrm{L}$ \\
\hline & Trichloroethene & $2.00 \mathrm{U}$ & $2.00 \mathrm{U}$ & & & $\mu \mathrm{g} / \mathrm{L}$ \\
\hline \multirow[t]{8}{*}{$X 749-45 G$} & 1,1,1-Trichloroethane & $2.00 \mathrm{U}$ & $2.00 \mathrm{U}$ & & & $\mu \mathrm{g} / \mathrm{L}$ \\
\hline & 1,1-Dichloroethane & $2.00 \mathrm{U}$ & $2.00 \mathrm{U}$ & & & $\mu \mathrm{g} / \mathrm{L}$ \\
\hline & 1,1-Dichloroethene & $2.00 \mathrm{U}$ & $2.00 \mathrm{U}$ & & & $\mu \mathrm{g} / \mathrm{L}$ \\
\hline & 1,2-Dichloroethane & $2.00 \mathrm{U}$ & $2.00 \mathrm{U}$ & & & $\mu \mathrm{g} / \mathrm{L}$ \\
\hline & 1,2-Dichloroethenes (cis \& $\mathrm{t}$ ) & $2.00 \mathrm{U}$ & $2.00 \mathrm{U}$ & & & $\mu \mathrm{g} / \mathrm{L}$ \\
\hline & Chloroform & $2.00 \mathrm{U}$ & $2.00 \mathrm{U}$ & & & $\mu \mathrm{g} / \mathrm{L}$ \\
\hline & Freon 113 & $2.00 \mathrm{U}$ & 3.00 & & & $\mu \mathrm{g} / \mathrm{L}$ \\
\hline & Trichloroethene & 4.00 & 8.00 & 5.33 & 2.31 & $\mu \mathrm{g} / \mathrm{L}$ \\
\hline \multirow[t]{3}{*}{ X749-PZ02G } & 1,1,1-Trichloroethane & $2.00 \mathrm{U}$ & $2.00 \mathrm{U}$ & & & $\mu \mathrm{g} / \mathrm{L}$ \\
\hline & 1,1-Dichloroethane & $2.00 \mathrm{U}$ & $2.00 \mathrm{U}$ & & & $\mu \mathrm{g} / \mathrm{L}$ \\
\hline & 1,1-Dichloroethene & $2.00 \mathrm{U}$ & $2.00 \mathrm{U}$ & & & $\mu \mathrm{g} / \mathrm{L}$ \\
\hline
\end{tabular}


Table 3.3. DOE/PORTS groundwater monitoring for 1995: results for volatile organic compounds at X-749 (continued)

\begin{tabular}{|c|c|c|c|c|c|c|}
\hline Well & Parameter & Min & $\operatorname{Max}$ & Mean & $\begin{array}{l}\text { Standard } \\
\text { Deviation }\end{array}$ & Units \\
\hline \multicolumn{7}{|c|}{ Gallia Wells (cont) } \\
\hline \multirow[t]{4}{*}{$\mathrm{X749-PZ02G}$} & 1,2-Dichloroethane & $2.00 \mathrm{U}$ & $2.00 \mathrm{U}$ & & & $\mu \mathrm{g} / \mathrm{L}$ \\
\hline & Chloroform & $2.00 \mathrm{U}$ & $2.00 \mathrm{U}$ & & & $\mu g / L$ \\
\hline & Freon 113 & $2.00 \mathrm{U}$ & $2.00 \mathrm{U}$ & & & $\mu \mathrm{g} / \mathrm{L}$ \\
\hline & Trichloroethene & $2.00 \mathrm{U}$ & $2.00 \mathrm{U}$ & & & $\mu \mathrm{g} / \mathrm{L}$ \\
\hline \multirow[t]{8}{*}{ X749-PZ03G } & 1,1,1-Trichloroethane & $2.00 \mathrm{U}$ & $2.00 \mathrm{U}$ & & & $\mu \mathrm{g} / \mathrm{L}$ \\
\hline & 1,1-Dichloroethane & $2.00 \mathrm{U}$ & $2.00 \mathrm{U}$ & & & $\mu \mathrm{g} / \mathrm{L}$ \\
\hline & 1,1-Dichloroethene & $2.00 \mathrm{U}$ & $2.00 \mathrm{U}$ & & & $\mu \mathrm{g} / \mathrm{L}$ \\
\hline & 1,2-Dichloroethane & $2.00 \mathrm{U}$ & $2.00 \mathrm{U}$ & & & $\mu \mathrm{g} / \mathrm{L}$ \\
\hline & 1,2-Dichloroethenes (cis \& t) & $2.00 \mathrm{U}$ & $2.00 \mathrm{U}$ & & & $\mu \mathrm{g} / \mathrm{L}$ \\
\hline & Chloroform & $2.00 \mathrm{U}$ & $2.00 \mathrm{U}$ & & & $\mu g / L$ \\
\hline & Freon 113 & $2.00 \mathrm{U}$ & $2.00 \mathrm{U}$ & & & $\mu \mathrm{g} / \mathrm{L}$ \\
\hline & Trichloroethene & $2.00 \mathrm{U}$ & $2.00 \mathrm{U}$ & & & $\mu \mathrm{g} / \mathrm{L}$ \\
\hline \multirow[t]{8}{*}{ X749-PZ04G } & 1,1,1-Trichloroethane & 18.00 & 21.00 & 19.25 & 1.26 & $\mu \mathrm{g} / \mathrm{L}$ \\
\hline & 1,1-Dichloroethane & 35.00 & 44.00 & 38.75 & 3.77 & $\mu \mathrm{g} / \mathrm{L}$ \\
\hline & 1,1-Dichloroethene & 19.00 & 22.00 & 19.75 & 1.50 & $\mu \mathrm{g} / \mathrm{L}$ \\
\hline & 1,2-Dichloroethane & 11.00 & 12.00 & 11.25 & 0.50 & $\mu \mathrm{g} / \mathrm{L}$ \\
\hline & 1,2-Dichloroethenes (cis \& t) & 11.00 & 11.00 & $11: 00$ & & $\mu \mathrm{g} / \mathrm{L}$ \\
\hline & Chloroform & 6.00 & 6.00 & 6.00 & 0.00 & $\mu \mathrm{g} / \mathrm{L}$ \\
\hline & Freon 113 & 46.00 & 51.00 & 47.75 & 2.36 & $\mu \mathrm{g} / \mathrm{L}$ \\
\hline & Trichloroethene & 130.00 & 150.00 & 135.00 & 10.00 & $\mu \mathrm{g} / \mathrm{L}$ \\
\hline \multirow[t]{8}{*}{ X749-PZ05G } & 1,1,1-Trichloroethane & $2.00 \mathrm{U}$ & $2.00 \mathrm{U}$ & 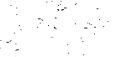 & & $\mu \mathrm{g} / \mathrm{L}$ \\
\hline & 1,1-Dichloroethane & $2.00 \mathrm{U}$ & $2.00 \mathrm{U}$ & & & $\mu \mathrm{g} / \mathrm{L}$ \\
\hline & 1,1-Dichloroethene & $2.00 \mathrm{U}$ & $2.00 \mathrm{U}$ & & & $\mu \mathrm{g} / \mathrm{L}$ \\
\hline & 1,2-Dichloroethane & $2.00 \mathrm{U}$ & $2.00 \mathrm{U}$ & & & $\mu \mathrm{g} / \mathrm{L}$ \\
\hline & 1,2-Dichloroethenes (cis \& t) & $2.00 \mathrm{U}$ & $2.00 \mathrm{U}$ & & & $\mu \mathrm{g} / \mathrm{L}$ \\
\hline & Chloroform & $2.00 \mathrm{U}$ & $2.00 \mathrm{U}$ & & & $\mu \mathrm{g} / \mathrm{L}$ \\
\hline & Freon 113 & $2.00 \mathrm{U}$ & $2.00 \mathrm{U}$ & & & $\mu \mathrm{g} / \mathrm{L}$ \\
\hline & Trichloroethene & $2.00 \mathrm{U}$ & $2.00 \mathrm{U}$ & & & $\mu \mathrm{g} / \mathrm{L}$ \\
\hline
\end{tabular}

Data Qualifer (U)--Parameter not detected. Result is attainable detection limit for the sample. Mean and standard deviation functions not calculated if at least $50 \%$ of the results for the parameter were below detection. 
Table 3.4. DOE/PORTS groundwater monitoring for 1995: results for radionuclide parameters at $X-749$

\begin{tabular}{|c|c|c|c|c|c|c|}
\hline Well & Parameter & Min & Max & Mean & $\begin{array}{l}\text { Standard } \\
\text { Deviation }\end{array}$ & Units \\
\hline \multicolumn{7}{|c|}{ Berea Wells (cont) } \\
\hline \multirow[t]{4}{*}{ X749-14B } & Gross Alpha & $4.00 \mathrm{U}$ & 5.00 & 4.86 & 0.38 & $\mathrm{pCi} / \mathrm{L}$ \\
\hline & Gross Beta & $11.00 \mathrm{U}$ & $11.00 \mathrm{U}$ & & & $\mathrm{pCi} / \mathrm{L}$ \\
\hline & Technetium & $22.00 \mathrm{U}$ & $22.00 \mathrm{U}$ & & & $\mathrm{pCi} / \mathrm{L}$ \\
\hline & Uranium & $1.00 \mathrm{U}$ & $1.00 \mathrm{U}$ & & & $\mu \mathrm{g} / \mathrm{L}$ \\
\hline \multirow[t]{4}{*}{ X749-50B } & Gross Alpha & $9.50 \mathrm{U}$ & 25.00 & & & $\mathrm{pCi} / \mathrm{L}$ \\
\hline & Gross Beta & $12.00 \mathrm{U}$ & 25.00 & & & $\mathrm{pCi} / \mathrm{L}$ \\
\hline & Technetium & $22.00 \mathrm{U}$ & $24.00 \mathrm{U}$ & & & $\mathrm{pCi} / \mathrm{L}$ \\
\hline & Uranium & 1.00 & 4.20 & 2.18 & 1.52 & $\mu \mathrm{g} / \mathrm{L}$ \\
\hline \multirow[t]{4}{*}{ X749-51B } & Gross Alpha & 5.00 & 7.00 & 6.00 & 1.15 & $\mathrm{pCi} / \mathrm{L}$ \\
\hline & Gross Beta & 9.00 & 14.00 & & & $\mathrm{pCi} / \mathrm{L}$ \\
\hline & Technetium & $22.00 \mathrm{U}$ & $24.00 \mathrm{U}$ & & & $\mathrm{pCi} / \mathrm{L}$ \\
\hline & Uranium & $1.00 \mathrm{U}$ & $1.00 \mathrm{U}$ & & & $\mu \mathrm{g} / \mathrm{L}$ \\
\hline \multirow[t]{4}{*}{ X749-54B } & Gross Alpha & 10.00 & 75.00 & 34.33 & 35.44 & $\mathrm{pCi} / \mathrm{L}$ \\
\hline & Gross Beta & 12.00 & 35.00 & 25.67 & 12.10 & $\mathrm{pCi} / \mathrm{L}$ \\
\hline & Technetium & $22.00 \mathrm{U}$ & $24.00 \mathrm{U}$ & & & $\mathrm{pCi} / \mathrm{L}$ \\
\hline & Uranium & $1.00 \mathrm{U}$ & $1.00 \mathrm{U}$ & & & $\mu \mathrm{g} / \mathrm{L}$ \\
\hline \multirow[t]{4}{*}{ X749-60B } & Gross Alpha & 18.00 & 23.00 & 19.67 & 2.89 & $\mathrm{pCi} / \mathrm{L}$ \\
\hline & Gross Beta & 12.00 & 17.00 & 14.33 & 2.52 & $\mathrm{pCi} / \mathrm{L}$ \\
\hline & Technetium & $22.00 \mathrm{U}$ & $24.00 \mathrm{U}$ & & & $\mathrm{pCi} / \mathrm{L}$ \\
\hline & Uranium & 1.30 & 1.50 & 1.43 & 0.12 & $\mu \mathrm{g} / \mathrm{L}$ \\
\hline \multirow[t]{5}{*}{ X749-64B } & Gross Alpha & $24.00 \mathrm{U}$ & 42.00 & 33.67 & 9.07 & $\mathrm{pCi} / \mathrm{L}$ \\
\hline & Gross Beta & 22.00 & $45.00 \mathrm{U}$ & & & $\mathrm{pCi} / \mathrm{L}$ \\
\hline & Technetium & $22.00 \mathrm{U}$ & $24.00 \mathrm{U}$ & & & $\mathrm{pCi} / \mathrm{L}$ \\
\hline & Uranium & 1.50 & 2.80 & 2.33 & 0.72 & $\mu \mathrm{g} / \mathrm{L}$ \\
\hline & & Gallia $W_{c}$ & & & & \\
\hline \multirow[t]{4}{*}{$\mathrm{X} 120-08 \mathrm{G}$} & Gross Alpha & $3.00 \mathrm{U}$ & 4.00 & & & $\mathrm{pCi} / \mathrm{L}$ \\
\hline & Gross Beta & $11.00 \mathrm{U}$ & $11.00 \mathrm{U}$ & & & $\mathrm{pCi} / \mathrm{L}$ \\
\hline & Technetium & $22.00 \mathrm{U}$ & $24.00 \mathrm{U}$ & & & $\mathrm{pCi} / \mathrm{L}$ \\
\hline & Uranium & 1.30 & 2.50 & 1.90 & 0.69 & $\mu \mathrm{g} / \mathrm{L}$ \\
\hline
\end{tabular}


Table 3.4. DOE/PORTS groundwater monitoring for 1995: results for radionuclide parameters at $X-749$ (continued)

\begin{tabular}{|c|c|c|c|c|c|c|}
\hline Well & Parameter & $\operatorname{Min}$ & Max & Mean & $\begin{array}{l}\text { Standard } \\
\text { Deviation }\end{array}$ & Units \\
\hline \multicolumn{7}{|c|}{ Gallia Wells (cont) } \\
\hline \multirow[t]{4}{*}{$\times 749-04 G$} & Gross Alpha & $7.00 \mathrm{U}$ & $9.00 \mathrm{U}$ & & & $\mathrm{pCi} / \mathrm{L}$ \\
\hline & Gross Beta & $8.00 \mathrm{U}$ & $8.00 \mathrm{U}$ & & & $\mathrm{pCi} / \mathrm{L}$ \\
\hline & Technetium & $22.00 \mathrm{U}$ & $24.00 \mathrm{U}$ & & & $\mathrm{pCi} / \mathrm{L}$ \\
\hline & Uranium & $1.00 \mathrm{U}$ & $1.00 \mathrm{U}$ & & & $\mu \mathrm{g} / \mathrm{L}$ \\
\hline \multirow[t]{4}{*}{ X749-06G } & Gross Alpha & $4.00 \mathrm{U}$ & 7.00 & & & $\mathrm{pCi} / \mathrm{L}$ \\
\hline & Gross Beta & 55.00 & 88.00 & 72.00 & 18.49 & $\mathrm{pCi} / \mathrm{L}$ \\
\hline & Technetium & 64.00 & 104.00 & 87.75 & 19.74 & $\mathrm{pCi} / \mathrm{L}$ \\
\hline & Uranium & $1.00 \mathrm{U}$ & $1.00 \mathrm{U}$ & & & $\mu \mathrm{g} / \mathrm{L}$ \\
\hline \multirow[t]{4}{*}{$X 749-07 \mathrm{G}$} & Gross Alpha & 7.00 & 12.00 & 9.00 & 2.65 & $\mathrm{pCi} / \mathrm{L}$ \\
\hline & Gross Beta & 152.00 & 305.00 & 208.67 & 83.86 & $\mathrm{pCi} / \mathrm{L}$ \\
\hline & Technetium & 214.00 & 407.00 & 281.00 & 109.19 & $\mathrm{pCi} / \mathrm{L}$ \\
\hline & Uranium & $1.00 \mathrm{U}$ & $1.00 \mathrm{U}$ & & & $\mu \mathrm{g} / \mathrm{L}$ \\
\hline \multirow[t]{4}{*}{$X 749-08 \mathrm{G}$} & Gross Alpha & $4.00 \mathrm{U}$ & $5.00 \mathrm{U}$ & & & $\mathrm{pCi} / \mathrm{L}$ \\
\hline & Gross Beta & 112.00 & 133.00 & 117.40 & 8.85 & $\mathrm{pCi} / \mathrm{L}$ \\
\hline & Technetium & 119.00 & 170.00 & 132.00 & 21.53 & $\mathrm{pCi} / \mathrm{L}$ \\
\hline & Uranium & $1.00 \mathrm{U}$ & $1.00 \mathrm{U}$ & & & $\mu \mathrm{g} / \mathrm{L}$ \\
\hline \multirow[t]{4}{*}{ X749-09G } & Gross Alpha & $5.00 \mathrm{U}$ & 26.00 & & & $\mathrm{pCi} / \mathrm{L}$ \\
\hline & Gross Beta & $9.00 \bar{U}$ & $11.20 \mathrm{U}$ & & & $\mathrm{pCi} / \mathrm{L}$ \\
\hline & Technetium & $22.00 \mathrm{U}$ & $24.00 \mathrm{U}$ & & & $\mathrm{pCi} / \mathrm{L}$ \\
\hline & Uranium & $1.00 \mathrm{U}$ & $1.00 \mathrm{U}$ & & & $\mu \mathrm{g} / \mathrm{L}$ \\
\hline \multirow[t]{4}{*}{ X749-10G } & Gross Alpha & $6.00 \mathrm{U}$ & $9.00 \mathrm{U}$ & & & $\mathrm{pCi} / \mathrm{L}$ \\
\hline & Gross Beta & $8.00 \mathrm{U}$ & 10.00 & & & $\mathrm{pCi} / \mathrm{L}$ \\
\hline & Technetium & $22.00 \mathrm{U}$ & $24.00 \mathrm{U}$ & & & $\mathrm{pCi} / \mathrm{L}$ \\
\hline & Uranium & 2.20 & 4.50 & 3.30 & 1.15 & $\mu \mathrm{g} / \mathrm{L}$ \\
\hline \multirow[t]{4}{*}{$X 749-13 G$} & Gross Alpha & $4.00 \mathrm{U}$ & 9.00 & & & $\mathrm{pCi} / \mathrm{L}$ \\
\hline & Gross Beta & 76.00 & 107.00 & 94.33 & $16: 26$ & $\mathrm{pCi} / \mathrm{L}$ \\
\hline & Technetium & 126.00 & 151.00 & 140.33 & 12.90 & $\mathrm{pCi} / \mathrm{L}$ \\
\hline & Uranium : & $1.00 \mathrm{U}$ & $1.00 \mathrm{U}$ & & & $\mu \mathrm{g} / \mathrm{L}$ \\
\hline
\end{tabular}


Table 3.4. DOE/PORTS groundwater monitoring for 1995:

results for radionuclide parameters at X-749 (continued)

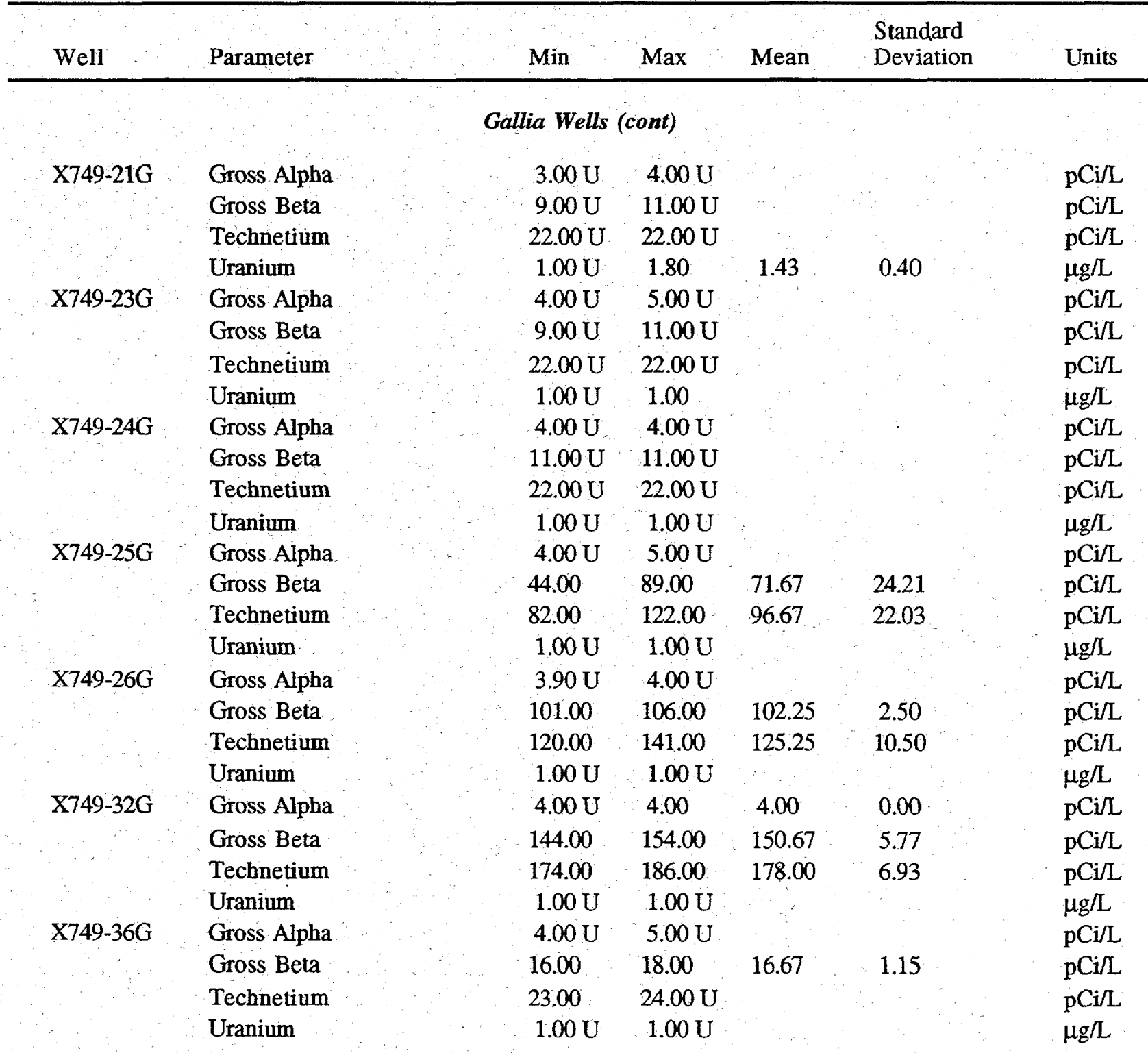


Table 3.4. DOE/PORTS groundwater monitoring for 1995: results for radionuclide parameters at $X-749$ (continued)

\begin{tabular}{|c|c|c|c|c|c|c|}
\hline Well & Parameter & Min & Max & Mean & $\begin{array}{l}\text { Standard } \\
\text { Deviation }\end{array}$ & Units \\
\hline \multicolumn{7}{|c|}{ Gallia Wells (cont) } \\
\hline \multirow[t]{4}{*}{ X749-37G } & Gross Alpha & $4.00 \mathrm{U}$ & $4.00 \mathrm{U}$ & & & $\mathrm{pC} \mathbf{i} / \mathrm{L}$ \\
\hline & Gross Beta & 21.00 & 27.00 & 23.67 & -3.06 & $\mathrm{pCi} / \mathrm{L}$ \\
\hline & Technetium & 22.00 & 42.00 & 29.33 & 11.02 & $\mathrm{pCi} / \mathrm{L}$ \\
\hline & Uranium & $1.00 \mathrm{U}$ & $1.00 \mathrm{U}$ & & & $\mu \mathrm{g} / \mathrm{L}$ \\
\hline \multirow[t]{4}{*}{$X 749-43 G$} & Gross Alpha & $4.00 \mathrm{U}$ & $4.00 \mathrm{U}$ & & & $\mathrm{pCi} / \mathrm{L}$ \\
\hline & Gross Beta & $8.00 \mathrm{U}$ & $9.00 \mathrm{U}$ & & & $\mathrm{PCi} / \mathrm{L}$ \\
\hline & Technetium & $22.00 \mathrm{U}$ & $24.00 \mathrm{U}$ & & & $\mathrm{pCi} / \mathrm{L}$ \\
\hline & Uranium & $1.00 \mathrm{U}$ & 2.80 & 1.97 & 0.91 & $\mu \mathrm{g} / \mathrm{L}$ \\
\hline \multirow[t]{4}{*}{$\times 749-44 G$} & Gross Alpha & $4.00 \mathrm{U}$ & 6.00 & & & $\mathrm{pCi} / \mathrm{L}$ \\
\hline & Gross Beta & $8.00 \mathrm{U}$ & $11.00 \mathrm{U}$ & & & $\mathrm{pCi} / \mathrm{L}$ \\
\hline & Technetium & $22.00 \mathrm{U}$ & $24.00 \mathrm{U}$ & & & $\mathrm{pCi} / \mathrm{L}$ \\
\hline & Uranium & $1.00 \mathrm{U}$ & 1.20 & & & $\mu \mathrm{g} / \mathrm{L}$ \\
\hline \multirow[t]{4}{*}{$\mathrm{X} 749-45 \mathrm{G}$} & Gross Alpha & $4.00 \mathrm{U}$ & $6.00 \mathrm{U}$ & & & $\mathrm{pCi} / \mathrm{L}$ \\
\hline & Gross Beta & $8.00 \mathrm{U}$ & $11.00 \mathrm{U}$ & & & $\mathrm{pCi} / \mathrm{L}$ \\
\hline & Technetium & $22.00 \mathrm{U}$ & $24.00 \mathrm{U}$ & & & $\mathrm{pCi} / \mathrm{L}$ \\
\hline & Uranium & $1.00 \mathrm{U}$ & 1.70 & 1.33 & 0.35 & $\mu \mathrm{g} / \mathrm{L}$ \\
\hline \multirow[t]{4}{*}{$X 749-P Z 02 G$} & Gross Alpha & $4.00 \mathrm{U}$ & $4.00 \mathrm{U}$ & & & $\mathrm{pCi} / \mathrm{L}$ \\
\hline & Gross Beta & $8.00 \mathrm{U}$ & $11.00 \mathrm{U}$ & & & $\mathrm{pCi} / \mathrm{L}$ \\
\hline & Technétium & $22.00 \mathrm{U}$ & $24.00 \mathrm{U}$ & & & $\mathrm{pCi} / \mathrm{L}$ \\
\hline & Uranium & $1.00 \mathrm{U}$ & $1.00 \mathrm{U}$ & & & $\mu \mathrm{g} / \mathrm{L}$ \\
\hline \multirow[t]{4}{*}{ X749-PZ03G } & Gross Alpha & $3.00 \mathrm{U}$ & $4.00 \mathrm{U}$ & & & $\mathrm{pCi} / \mathrm{L}$ \\
\hline & Gross Beta & $8.00 \mathrm{U}$ & $11.00 \mathrm{U}$ & & & $\mathrm{pCi} / \mathrm{L}$ \\
\hline & Technetium & $22.00 \mathrm{U}$ & $24.00 \mathrm{U}$ & & & $\mathrm{pCi} / \mathrm{L}$ \\
\hline & Uranium & $1.00 \mathrm{U}$ & 1.00 & & & $\mu \mathrm{g} / \mathrm{L}$ \\
\hline \multirow[t]{4}{*}{ X749-PZ04G } & Gross Alpha & $4.00 \mathrm{U}$ & $5.00 \mathrm{U}$ & & & $\mathrm{pCi} / \mathrm{L}$ \\
\hline & Gross Beta & 75.00 & 89.00 & 78.75 & 6.85 & $\mathrm{pCi} / \mathrm{L}$ \\
\hline & Technetium & 101.00 & 134.00 & 114.75 & 13.84 & $\mathrm{pCi} / \mathrm{L}$ \\
\hline & Uranium & $1.00 \mathrm{U}$ & $1.00 \mathrm{U}$ & & & $\mu \mathrm{g} / \mathrm{L}$ \\
\hline
\end{tabular}


Table 3.4. DOE/PORTS groundwater monitoring for 1995: results for radionuclide parameters at X-749 (continued)

\begin{tabular}{|c|c|c|c|c|c|c|}
\hline Well & Parameter & Min & Max & Mean & $\begin{array}{l}\text { Standard } \\
\text { Deviation }\end{array}$ & Units \\
\hline \multicolumn{7}{|c|}{ Gallia Wells (cont) } \\
\hline \multirow[t]{4}{*}{$\mathrm{X749-PZ05G}$} & Gross Alpha & $5.00 \mathrm{U}$ & 9.00 & 6.33 & 2.31 & $\mathrm{pCi} / \mathrm{L}$ \\
\hline & Gross Beta & $8.00 \mathrm{U}$ & $11.00 \mathrm{U}$ & & & $\mathrm{pCi} / \mathrm{L}$ \\
\hline & Technetium & $22.00 \mathrm{U}$ & $24.00 \mathrm{U}$ & & & $\mathrm{pCi} / \mathrm{L}$ \\
\hline & Uranium & 5.60 & 9.10 & 6.83 & 1.97 & $\mu \mathrm{g} / \mathrm{L}$ \\
\hline
\end{tabular}

Data Qualifer (U)--Parameter not detected. Result is attainable detection limit for the sample. Mean and standard deviation functions not calculated if at least $50 \%$ of the results for the parameter were below detection. 
Table 3.5. DOE/PORTS groundwater monitoring for 1995: results for volatile organic compounds at $\mathrm{X}-231 \mathrm{~B}$

Well Parameter Min Max Mean Deviation Units

\section{Berea Wells}

\begin{tabular}{|c|c|c|c|c|}
\hline \multirow[t]{3}{*}{$\mathrm{X} 231 \mathrm{~B}-24 \mathrm{~B}$} & $1,1,1$-Trichloroethane & $2.00 \mathrm{U}$ & $2.00 \mathrm{U}$ & $\mu \mathrm{g} / \mathrm{L}$ \\
\hline & 1,1-Dichloroethene & $2.00 \mathrm{U}$ & $2.00 \mathrm{U}$ & $\mu g / L$ \\
\hline & Trichloroethene & $2.00 \mathrm{U}$ & $2.00 \mathrm{U}$ & $\mu \mathrm{g} / \mathrm{L}$ \\
\hline \multirow[t]{3}{*}{$\mathrm{X} 231 \mathrm{~B}-32 \mathrm{~B}$} & 1,1,1-Trichloroethane & $2.00 \mathrm{U}$ & $2.00 \mathrm{U}$ & \\
\hline & 1,1-Dichloroethene & $2.00 \mathrm{U}$ & $2.00 \mathrm{U}$ & \\
\hline & Trichloroethene & $2.00 \mathrm{U}$ & $2.00 \mathrm{U}$ & \\
\hline \multirow[t]{3}{*}{ X231B-33B } & 1,1,1-Trichloroethane & $2.00 \mathrm{U}$ & $2.00 \mathrm{U}$ & \\
\hline & 1,1-Dichloroethene & $2.00 \mathrm{U}$ & $2.00 \mathrm{U}$ & \\
\hline & Trichloroethene & $2.00 \mathrm{U}$ & $2.00 \mathrm{U}$ & \\
\hline \multirow[t]{3}{*}{$\mathrm{X} 231 \mathrm{~B}-34 \mathrm{~B}$} & 1,1,1-Trichloroethane & $2.00 \mathrm{U}$ & $2.00 \mathrm{U}$ & \\
\hline & 1,1-Dichloroethene & $2.00 \mathrm{U}$ & $2.00 \mathrm{U}$ & \\
\hline & Trichloroethene & $2.00 \mathrm{U}$ & $2.00 \mathrm{U}$ & \\
\hline
\end{tabular}

Gallia Wells

\begin{tabular}{|c|c|c|c|c|c|c|}
\hline X231B-02G & 1,1,1-Trichloroethane & 82.00 & $200.00 \mathrm{U}$ & & & $\mu g / L$ \\
\hline & 1,1-Dichloroethene & 48.00 & $200.00 \mathrm{U}$ & & & $\mu \mathrm{g} / \mathrm{L}$ \\
\hline & Trichloroethene & 1600.00 & 2400.00 & 1966.67 & 404.15 & T. \\
\hline $\mathrm{X} 231 \mathrm{~B}-03 \mathrm{G}$ & 1,1,1-Trichloroethane & .120 .00 & $200.00 \mathrm{U}$ & 153.33 & 41.63 & \\
\hline & 1,1-Dichloroethene & 110.00 & $200.00 \mathrm{U}$ & 143.33 & 49.33 & \\
\hline & Trichloroethene & 600.00 & 760.00 & 673.33 & 80.83 & \\
\hline $\mathrm{X} 231 \mathrm{~B}-04 \mathrm{G}$ & 1,1,1-Trichloroethane & $20.00 \mathrm{U}$ & $20.00 \mathrm{U}$ & & & \\
\hline & 1,1-Dichloroethene & $20.00 \mathrm{U}$ & $20.00 \mathrm{U}$ & & & \\
\hline & Trichloroethene & 360.00 & 610.00 & 496.67 & 126.62 & \\
\hline $\mathrm{X} 231 \mathrm{~B}-06 \mathrm{G}$ & 1,1,1-Trichloroethane & 160.00 & 180.00 & 170.00 & 10.00 & \\
\hline & 1,1-Dichloroethene & $20.00 \mathrm{U}$ & 130.00 & 83.33 & 56.86 & \\
\hline & Trichloroethene & 160.00 & 240.00 & 193.33 & 41.63 & \\
\hline $231 B-14 G$ & 1,1,1-Trichloroethane & $2.00 \mathrm{U}$ & $2.00 \mathrm{U}$ & & & \\
\hline & 1,1-Dichloroethene & $2.00 \mathrm{U}$ & $2.00 \mathrm{U}$ & & & \\
\hline & Trichloroethene & 41.00 & 49.00 & 44.80 & 3.63 & \\
\hline
\end{tabular}


Table 3.5. DOE/PORTS groundwater monitoring for 1995: results for volatile organic compounds at X-231B (continued)

\begin{tabular}{|c|c|c|c|c|c|c|}
\hline Well & Parameter & Min & $\operatorname{Max}$ & Mean & $\begin{array}{l}\text { Standard } \\
\text { Deviation }\end{array}$ & Units \\
\hline & & \multicolumn{2}{|c|}{ Gallia Wells (cont) } & & & \\
\hline \multirow[t]{3}{*}{$\mathrm{X} 231 \mathrm{~B}-15 \mathrm{G}$} & 1,1,1-Trichloroethane & $2.00 \mathrm{U}$ & $2.00 \mathrm{U}$ & & & $\mu g / L$ \\
\hline & 1,1-Dichloroethene & $2.00 \mathrm{U}$ & $2.00 \mathrm{U}$ & & & $\mu \mathrm{g} / \mathrm{L}$ \\
\hline & Trichloroethene & 5.00 & 85.00 & 59.8 & 31.36 & $\mu \mathrm{g} / \mathrm{L}$ \\
\hline \multirow[t]{3}{*}{$\mathrm{X} 231 \mathrm{~B}-16 \mathrm{G}$} & 1,1,1-Trichloroethane & $2.00 \mathrm{U}$ & $2.00 \mathrm{U}$ & & & $\mu \mathrm{g} / \mathrm{L}$ \\
\hline & 1,1-Dichloroethene & $2.00 \mathrm{U}$ & $2.00 \mathrm{U}$ & & & $\mu \mathrm{g} / \mathrm{L}$ \\
\hline & Trichloroethene & $2.00 \mathrm{U}$ & $2.00 \mathrm{U}$ & & & $\mu \mathrm{g} / \mathrm{L}$ \\
\hline \multirow[t]{3}{*}{ X231B-23G } & 1,1,1-Trichloroethane & $2.00 \mathrm{U}$ & $2.00 \mathrm{U}$ & & & $\mu \mathrm{g} / \mathrm{L}$ \\
\hline & 1,1-Dichloroethene & $2.00 \mathrm{U}$ & $2.00 \mathrm{U}$ & & & $\mu \mathrm{g} / \mathrm{L}$ \\
\hline & Trichloroethene & 21.00 & 26.00 & 23.40 & 1.82 & $\mu g / L$ \\
\hline \multirow[t]{3}{*}{$\mathrm{X} 231 \mathrm{~B}-27 \mathrm{G}$} & 1,1,1-Trichloroethane & $2.00 \mathrm{U}$ & $2.00 \mathrm{U}$ & & & $\mu g / L$ \\
\hline & 1,1-Dichloroethene & $2.00 \mathrm{U}$ & $2.00 \mathrm{U}$ & & & $\mu \mathrm{g} / \mathrm{L}$ \\
\hline & Trichloroethene & $2.00 \mathrm{U}$ & $2.00 \mathrm{U}$ & & & $\mu \mathrm{g} / \mathrm{L}$ \\
\hline \multirow[t]{3}{*}{ X231B-28G } & 1,1,1-Trichloroethane & $2.00 \mathrm{U}$ & $2.00 \mathrm{U}$ & & & $\mu \mathrm{g} / \mathrm{L}$ \\
\hline & 1,1-Dichloroethene & $2.00 \mathrm{U}$ & $2.00 \mathrm{U}$ & & & $\mu \mathrm{g} / \mathrm{L}$ \\
\hline & Trichloroethene & $2.00 \mathrm{U}$ & $2.00 \mathrm{U}$ & & & $\mu \mathrm{g} / \mathrm{L}$ \\
\hline \multirow[t]{3}{*}{$\mathrm{X} 231 \mathrm{~B}-37 \mathrm{G}$} & 1,1,1-Trichloroethane & $2.00 \mathrm{U}$ & $2.00 \mathrm{U}$ & & & $\mu \mathrm{g} / \mathrm{L}$ \\
\hline & 1,1-Dichloroethene & 8.00 & 10.00 & 8.60 & 0.89 & $\mu \mathrm{g} / \mathrm{L}$ \\
\hline & Trichloroethene & 64.00 & 70.00 & 67.80 & 2.28 & $\mu \mathrm{g} / \mathrm{L}$ \\
\hline
\end{tabular}

Data Qualifer (U)--Parameter not detected. Result is attainable detection limit for the sample. Mean and standard deviation functions not calculated if at least $50 \%$ of the results for the parameter were below detection. 
Table 3.6. DOE/PORTS groundwater monitoring for 1995: results for radionuclide parameters at $\mathrm{X}-231 \mathrm{~B}$

\begin{tabular}{|c|c|c|c|c|c|c|}
\hline Well & Parameter & Min & $\operatorname{Max}$ & Mean & $\begin{array}{l}\text { Standard } \\
\text { Deviation }\end{array}$ & Units \\
\hline \multicolumn{7}{|c|}{ Berea Wells } \\
\hline $\mathrm{X} 231 \mathrm{~B}-24 \mathrm{~B}$ & Gross Alpha & 13.00 & $17.00 \mathrm{U}$ & & & $\mathrm{pCi} / \mathrm{L}$ \\
\hline & Gross Beta & $12.00 \mathrm{U}$ & 21.00 & & & $\mathrm{pCi} / \mathrm{I}$ \\
\hline & Technetium & $22.00 \mathrm{U}$ & $24.00 \mathrm{U}$ & & & $\mathrm{pCi} / \mathrm{I}$ \\
\hline & Uranium & 1.00 & 1.50 & 1.31 & 0.23 & $\mu \mathrm{g} / \mathrm{L}$ \\
\hline $\mathrm{X} 231 \mathrm{~B}-32 \mathrm{~B}$ & Gross Alpha & 13.00 & $23.00 \mathrm{U}$ & & & $\mathrm{pCi} / \mathrm{L}$ \\
\hline & Gross Beta & $1670 \mathrm{U}$ & $32.00 \mathrm{U}$ & & & $\mathrm{pCi} / \mathrm{L}$ \\
\hline & Technetium & $22.00 \mathrm{U}$ & $22.00 \mathrm{U}$ & & & $\mathrm{pCi} / \mathrm{L}$ \\
\hline & Uranium & $1.00 \mathrm{U}$ & 3.20 & & & $\mu \mathrm{g} / \mathrm{L}$ \\
\hline $\mathrm{X} 231 \mathrm{~B}-33 \mathrm{~B}$ & Gross Alpha & $10.00 \mathrm{U}$ & 11.00 & & & $\mathrm{pCi} / \mathrm{I}$ \\
\hline & Gross Beta & $12.00 \mathrm{U}$ & 17.00 & 2.71 & 1.89 & $\mathrm{pCi} / \mathrm{L}$ \\
\hline & Technetium & $22.00 \mathrm{U}$ & $24.00 \mathrm{U}$ & & & $\mathrm{pCi} / \mathrm{L}$ \\
\hline & Uranium & $1.00 \mathrm{U}$ & $1.00 \mathrm{U}$ & & & $\mu \mathrm{g} / \mathrm{L}$ \\
\hline $\mathrm{X} 231 \mathrm{~B}-34 \mathrm{~B}$ & Gross Alpha & 19.00 & 28.00 & 25.43 & 3.55 & $\mathrm{pCi} / \mathrm{L}$ \\
\hline & Gross Beta & 9.00 & 18.00 & 13.57 & 2.70 & $\mathrm{pCi} / \mathrm{L}$ \\
\hline & Technetium & $22.00 \mathrm{U}$ & $24.00 \mathrm{U}$ & & 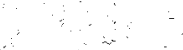 & $\mathrm{pCi} / \mathrm{L}$ \\
\hline & Uranium & 1.50 & 3.20 & 2.76 & 0.65 & $\mu \mathrm{g} / \mathrm{L}$ \\
\hline
\end{tabular}

Gallia Wells

\begin{tabular}{|c|c|c|c|c|c|c|}
\hline \multirow[t]{4}{*}{$\mathrm{X} 231 \mathrm{~B}-02 \mathrm{G}$} & Gross Alpha & $5.00 \mathrm{U}$ & $5.00 \mathrm{U}$ & & & $\mathrm{pCi} / \mathrm{L}$ \\
\hline & Gross Beta & $11.00 \mathrm{U}$ & 14.00 & 12.00 & 1.73 & $\mathrm{pCi} / \mathrm{L}$ \\
\hline & Technetium & $22.00 \mathrm{U}$ & $24.00 \mathrm{U}$ & & & $\mathrm{pCi} / \mathrm{L}$ \\
\hline & Uranium & $1.00 \mathrm{U}$ & $1.00 \mathrm{U}$ & & & $\mu \mathrm{g} / \mathrm{L}$ \\
\hline \multirow[t]{4}{*}{ X231B-03G } & Gross Alpha & $4.00 \mathrm{U}$ & $4.00 \mathrm{U}$ & & & $\mathrm{pCi} / \mathrm{L}$ \\
\hline & Gross Beta & $8.00 \mathrm{U}$. & $11.00 \mathrm{U}$ & & & $\mathrm{pCi} / \mathrm{L}$ \\
\hline & Technetium & $22.00 \mathrm{U}$ & $24.00 \mathrm{U}$ & & & $\mathrm{pCj} / \mathrm{L}$ \\
\hline & Uranium & $1.00 \mathrm{U}$ & $1.00 \mathrm{U}$ & & & $\mu g / L$ \\
\hline \multirow[t]{2}{*}{$\mathrm{X} 231 \mathrm{~B}-04 \mathrm{G}$} & Gross Alpha & $6.00 \mathrm{U}$ & 29.00 & & & $\mathrm{pCi}$ \\
\hline & Gross Beta & $11.00 \mathrm{U}$ & 19.00 & 16.33 & 4.62 & $\mathrm{C} \mathrm{i}$ \\
\hline
\end{tabular}


Table 3.6. DOE/PORTS groundwater monitoring for 1995:

results for radionuclide parameters at X-231B (continued)

\begin{tabular}{|c|c|c|c|c|c|c|}
\hline Well & Parameter & Min & Max & Mean & $\begin{array}{l}\text { Standard } \\
\text { Deviation }\end{array}$ & Ũnits \\
\hline \multicolumn{7}{|c|}{ Gallia Wells (cont) } \\
\hline X231B-04G & Technetium & $22.00 \mathrm{U}$ & 27.00 & & & $\mathrm{pCi} / \mathrm{L}$ \\
\hline & Uranium & 2.40 & 4.90 & 3.53 & 1.27 & $\mu \mathrm{g} / \mathrm{L}$ \\
\hline X231B-06G & Gross Alpha & 8.00 & 27.00 & 16.67 & 9.61 & $\mathrm{pCi} / \mathrm{L}$ \\
\hline & Gross Beta & 162.00 & 441.00 & 267.33 & 151.53 & $\mathrm{pC} / \mathrm{L}$ \\
\hline & Technetium & 229.00 & 551.00 & 345.00 & 178.87 & $\mathrm{pCi} / \mathrm{L}$ \\
\hline & Uranium & 2.20 & 4.60 & 3.00 & 1.39 & $\mu \mathrm{g} / \mathrm{L}$ \\
\hline $\mathrm{X} 231 \mathrm{~B}-14 \mathrm{G}$ & Gross Alpha & $3.90 \mathrm{U}$ & $4.00 \mathrm{U}$ & & & $\mathrm{pCi} / \mathrm{L}$ \\
\hline & Gross Beta & $8.00 \mathrm{U}$ & 26.00 & & & pCilL \\
\hline & Technetium & $22.00 \mathrm{U}$ & 33.00 & & & $\mathrm{pCi} / \mathrm{L}$ \\
\hline & Uranium & $1.00 \mathrm{U}$ & $1.00 \mathrm{U}$ & & & $\mu \mathrm{g} / \mathrm{L}$ \\
\hline $\mathrm{X} 231 \mathrm{~B}-15 \mathrm{G}$ & Gross Alpha & $8.00 \mathrm{U}$ & $9.00 U$ & & & $\mathrm{pCi} / \mathrm{L}$ \\
\hline & Gross Beta & $8.00 \mathrm{U}$ & $12.00 \mathrm{U}$ & & & $\mathrm{pCi} / \mathrm{L}$ \\
\hline & Technetium & $22.00 \mathrm{U}$ & $24.00 \mathrm{U}$ & & & $\mathrm{pCi} / \mathrm{L}$ \\
\hline & Uranium & $1.00 \mathrm{U}$ & $1.00 \mathrm{U}$ & & & $\mu \mathrm{g} / \mathrm{L}$ \\
\hline $\mathrm{X} 231 \mathrm{~B}-16 \mathrm{G}$ & Gross Alpha & $5.00 \mathrm{U}$ & $21.00 \mathrm{U}$ & & & pCil \\
\hline & Gross Beta & $8.80 \mathrm{U}$ & $32.00 \mathrm{U}$ & & & pCiL \\
\hline & Technetium & $22.00 \mathrm{U}$ & $22.00 \mathrm{U}$ & & & $\mathrm{pCi} / \mathrm{L}$ \\
\hline & Uranium & $1.00 \mathrm{U}$ & $1.00 \mathrm{U}$ & & & $\mu \mathrm{g} / \mathrm{L}$ \\
\hline $\mathrm{X} 231 \mathrm{~B}-23 \mathrm{G}$ & Gross Alpha & 6.00 & $9.00 \mathrm{U}$ & & & $\mathrm{pCi} / \mathrm{L}$ \\
\hline & Gross Beta & $8.00 \mathrm{U}$ & 15.00 & & & $\mathrm{pCi} / \mathrm{L}$ \\
\hline & Technetium & $22.00 \mathrm{U}$ & $24.00 \mathrm{U}$ & & & $\mathrm{pCi} / \mathrm{L}$ \\
\hline & Uranium & $1.00 \mathrm{U}$ & 2.50 & & & $\mu \mathrm{g} / \mathrm{L}$ \\
\hline $\mathrm{X} 231 \mathrm{~B}-27 \mathrm{G}$ & Gross Alpha & $3.90 \mathrm{U}$ & $4.00 \mathrm{U}$ & & & pCiL \\
\hline & Gross Beta & $8.00 \mathrm{U}$ & $8.00 \mathrm{U}$ & & & pCiL \\
\hline & Technetium & $22.00 \mathrm{U}$ & $22.00 \mathrm{U}$ & & & $\mathrm{pCi} / \mathrm{L}$ \\
\hline & Uranium & $1.00 \mathrm{U}$ & 1.20 & 1.11 & 0.11 & $\mu \mathrm{g} / \mathrm{L}$ \\
\hline $\mathrm{X} 231 \mathrm{~B}-28 \mathrm{G}$ & Gross Alpha & $8.80 \mathrm{U}$ & $9.00 \mathrm{U}$ & & & pCilL \\
\hline & Gross Beta & $8.00 \mathrm{U}$ & $12.00 \mathrm{U}$ & & & pCilL \\
\hline & Technetium & $22.00 \mathrm{U}$ & $24.00 \mathrm{U}$ & & & $\mathrm{pCi} / \mathrm{L}$ \\
\hline
\end{tabular}


Table 3.6. DOE/PORTS groundwater monitoring for 1995:

results for radionuclide parameters at $\mathrm{X}-231 \mathrm{~B}$ (continued)

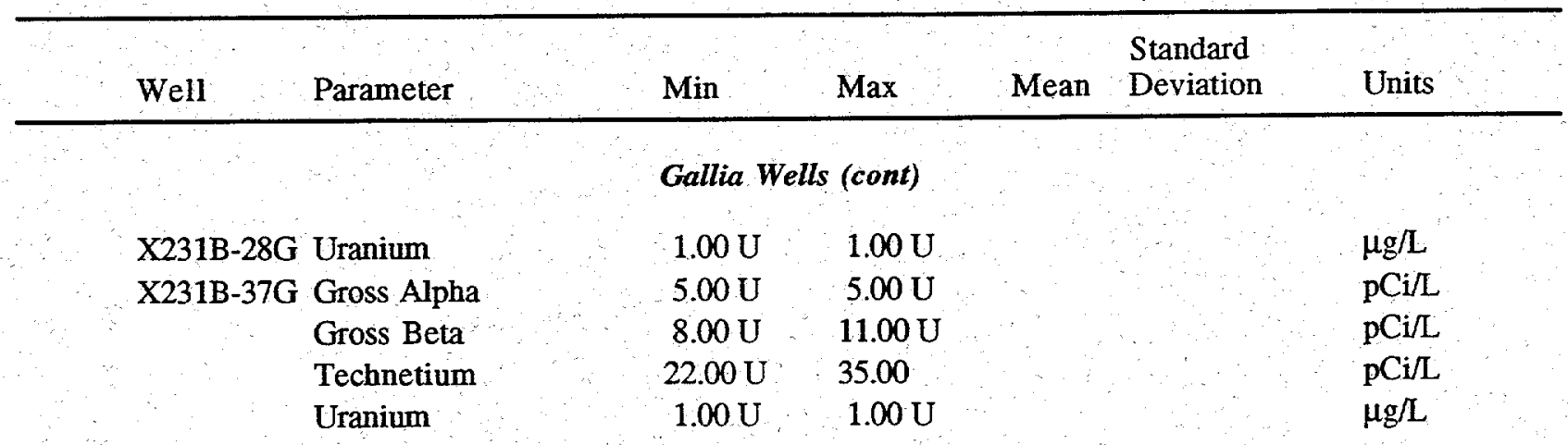

Data Qualifer (U)--Parameter not detected. Result is attainable detection limit for the sample. Mean and standard deviation functions not calculated if at least $50 \%$ of the results for the parameter were below detection. 
Table 3.7. DOE/PORTS groundwater monitoring for 1995: results for volatile organic compounds at X-616

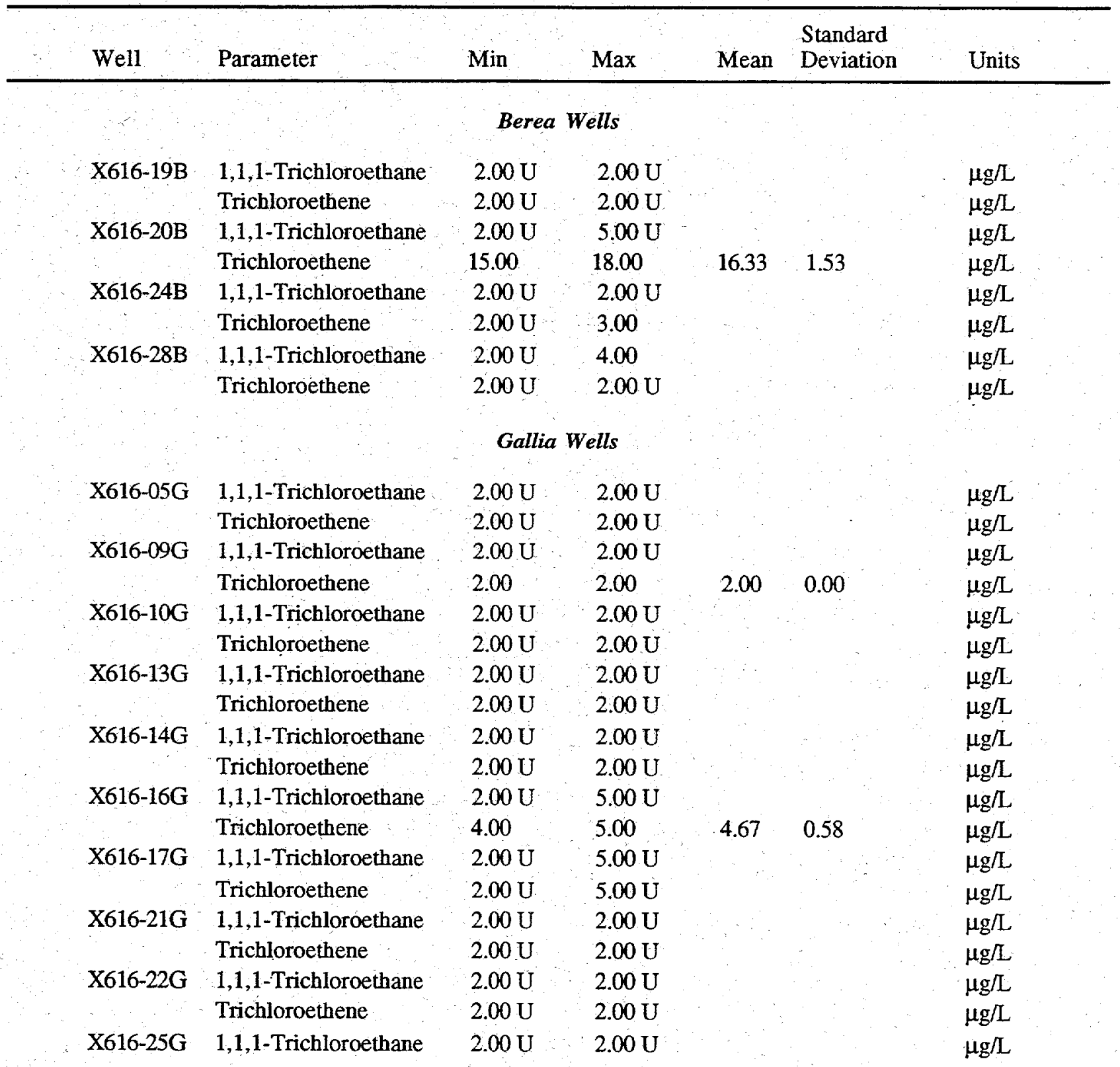


Table 3.7. DOEJPOATS groundwater monitoring for 1995: results for volatile organic compounds at X-616 (continued)

\begin{tabular}{|c|c|c|c|c|c|c|}
\hline Well & Parameter & Min & $\operatorname{Max}$ & Mean & $\begin{array}{l}\text { Standard } \\
\text { Deviation }\end{array}$ & Un \\
\hline \multicolumn{7}{|c|}{ Gallia Wells (cont) } \\
\hline X616-25G & Trichloroethene & $2.00 \mathrm{U}$ & $2.00 \mathrm{U}$ & & & $\mu \mathrm{g} / \mathrm{L}$ \\
\hline $\mathrm{X} 616-26 \mathrm{G}$ & 1,1,1-Trichloroethane & $2.00 \mathrm{U}$ & $2.00 \mathrm{U}$ & & & $\mu \mathrm{g}$ \\
\hline & Trichloroethene & $2.00 \mathrm{U}$ & $2.00 \mathrm{U}$ & & & $\mu \mathrm{g} /$ \\
\hline
\end{tabular}

Data Qualifer $(U)$--Parameter not detected. Result is attainable detection limit for the sample. Mean and standard deviation functions not calculated if at least $50 \%$ of the results for the parameter were below detection. 
Table 3.8. DOE/PORTS groundwater monitoring for 1995: results for dissolved and total chromium at X-616

\begin{tabular}{|c|c|c|c|c|c|c|}
\hline Well & Parameter & Min & Max & Mean & $\begin{array}{l}\text { Standard } \\
\text { Deviation }\end{array}$ & Units \\
\hline \multicolumn{7}{|c|}{ Berea Wells } \\
\hline \multirow[t]{2}{*}{ X616-19B } & Chromium (Dissolved) & $2.20 \mathrm{U}$ & $25.80 \mathrm{U}$ & & & $\mu \mathrm{g} / \mathrm{L}$ \\
\hline & Chromium (Total) & 25.20 & $25.80 \mathrm{U}$ & & & $\mu \mathrm{g} / \mathrm{L}$ \\
\hline \multirow[t]{2}{*}{$\mathrm{X} 616-20 \mathrm{~B}$} & Chromium (Dissolved) & $2.20 \mathrm{U}$ & $25.80 \mathrm{U}$ & & & $\mu \mathrm{g} / \mathrm{L}$ \\
\hline & Chromium (Total) & 7.10 & $25.80 \mathrm{U}$ & & & $\mu \mathrm{g} / \mathrm{L}$ \\
\hline \multirow[t]{2}{*}{$\mathrm{X} 616-24 \mathrm{~B}$} & Chromium (Dissolved) & 7.10 & $25.80 \mathrm{U}$ & & & $\mu \mathrm{g} / \mathrm{L}$ \\
\hline & Chromium (Total) & 14.30 & $25.80 \mathrm{U}$ & & & $\mu \mathrm{g} / \mathrm{L}$ \\
\hline \multirow[t]{2}{*}{$\mathrm{X} 616-28 \mathrm{~B}$} & Chromium (Dissolved) & 3.00 & $25.80 \mathrm{U}$ & & & $\mu \mathrm{g} / \mathrm{L}$ \\
\hline & Chromium (Total) & $2.20 \mathrm{U}$ & $25.80 \mathrm{U}$ & & & $\mu \mathrm{g} / \mathrm{L}$ \\
\hline \multicolumn{7}{|c|}{ Gallia Wells } \\
\hline \multirow[t]{2}{*}{$\mathrm{X} 616-05 \mathrm{G}$} & Chromium (Dissolved) & 124.00 & 236.00 & 180.00 & 79.20 & $\mu \mathrm{g} / \mathrm{L}$ \\
\hline & Chromium (Total) & 266.00 & 3840.00 & 2053.00 & 2527.20 & $\mu \mathrm{g} / \mathrm{L}$ \\
\hline \multirow[t]{2}{*}{$\mathrm{X} 616-09 \mathrm{G}$} & Chromium (Dissolved) & $2.20 \mathrm{U}$ & $25.80 \mathrm{U}$ & & & $\mu \mathrm{g} / \mathrm{L}$ \\
\hline & Chromium (Total) & 2.40 & $25.80 \mathrm{U}$ & & & $\mu \mathrm{g} / \mathrm{L}$ \\
\hline \multirow[t]{2}{*}{ X616-10G } & Chromium (Dissolved) & $2.20 \mathrm{U}$ & $25.80 \mathrm{U}$ & & & $\mu \mathrm{g} / \mathrm{L}$ \\
\hline & Chromium (Total) & $2.20 \mathrm{U}$ & $25.80 \mathrm{U}$ & & & $\mu \mathrm{g} / \mathrm{L}$ \\
\hline \multirow[t]{2}{*}{$\mathrm{X} 616-13 \mathrm{G}$} & Chromium (Dissolved) & $4.40 \mathrm{U}$ & $25.80 \mathrm{U}$ & & & $\mu \mathrm{g} / \mathrm{L}$ \\
\hline & Chromium (Total) & $2.20 \mathrm{U}$ & $25.80 \mathrm{U}$ & & & $\mu \mathrm{g} / \mathrm{L}$ \\
\hline \multirow[t]{2}{*}{ X616-14G } & Chromium (Dissolved) & $4.40 \mathrm{U}$ & $125.00 \mathrm{U}$ & & & $\mu \mathrm{g} / \mathrm{L}$ \\
\hline & Chromium (Total) & $4.40 \mathrm{U}$ & $125.00 \mathrm{U}$ & & & $\mu \mathrm{g} / \mathrm{L}$ \\
\hline \multirow[t]{2}{*}{ X616-16G } & Chromium (Dissolved) & $2.20 \mathrm{U}$ & $25.80 \mathrm{U}$ & & & $\mu \mathrm{g} / \mathrm{L}$ \\
\hline & Chromium (Total) & $2.20 \mathrm{U}$ & 60.00 & & & $\mu \mathrm{g} / \mathrm{L}$ \\
\hline \multirow[t]{2}{*}{$\mathrm{X} 616-17 \mathrm{G}$} & Chromium (Dissolved) & $2.20 \mathrm{U}$ & $25.80 \mathrm{U}$ & & & $\mu \mathrm{g} / \mathrm{L}$ \\
\hline & Chromium (Total) & 48.40 & 64.60 & 56.50 & 11.46 & $\mu \mathrm{g} / \mathrm{L}$ \\
\hline \multirow[t]{2}{*}{$\mathrm{X} 616-21 \mathrm{G}$} & Chromium (Dissolved) & 3.50 & $12.50 \mathrm{U}$ & & & $\mu \mathrm{g} / \mathrm{L}$ \\
\hline & Chromium (Total) & 7.10 & $12.50 \mathrm{U}$ & & & $\mu \mathrm{g} / \mathrm{L}$ \\
\hline \multirow[t]{2}{*}{$\mathrm{X} 616-22 \mathrm{G}$} & Chromium (Dissolved) & 5.50 & $12.50 \mathrm{U}$ & & & $\mu \mathrm{g} / \mathrm{L}$ \\
\hline & Chromium (Total) & 11.20 & 18.70 & 14.95 & 5.30 & $\mu \mathrm{g} / \mathrm{L}$ \\
\hline \multirow[t]{2}{*}{$\mathrm{X} 616-25 \mathrm{G}$} & Chromium (Dissolved) & $2.20 \mathrm{U}$ & $22.40 \mathrm{U}$ & & & $\mu \mathrm{g} / \mathrm{L}$ \\
\hline & Chromium (Total) & 21.40 & 76.20 & 48.80 & 38.75 & $\mu \mathrm{g} / \mathrm{L}$ \\
\hline
\end{tabular}


Table 3.8. DOE/PORTS groundwater monitoring for 1995: results for dissolved and total chromium at X-616 (continued)

\begin{tabular}{lll}
\hline Well Mn Marameter Man Deviation Units \\
\hline
\end{tabular}

Gallia Wells (cont)

\begin{tabular}{|c|c|c|}
\hline$G$ & $\begin{array}{l}\text { Chromium (Dissolved) } \\
\text { Chromium (Total) }\end{array}$ & $\begin{array}{l}4.40 \mathrm{U} \\
4.40 \mathrm{U}\end{array}$ \\
\hline
\end{tabular}

Data Qualifer (U)--Parameter not detected. Result is attainable detection limit for the sample. Mean and standard deviation functions not calculated if at least $50 \%$ of the results for the parameter were below detection. 
Table 3.9. DOE/PORTS groundwater monitoring for 1995:

results for radionuclide parameters at surface water monitoring locations

\begin{tabular}{|c|c|c|c|c|c|c|}
\hline Well & Parameter & Min & Max & Mean & $\begin{array}{l}\text { Standard } \\
\text { Deviation }\end{array}$ & Units \\
\hline \multirow[t]{4}{*}{ BRCSW01 } & Gross Alpha & $5.00 \mathrm{U}$ & $11.00 \mathrm{U}$ & & & $\mathrm{pCi} / \mathrm{L}$ \\
\hline & Gross Beta & $8.00 \mathrm{U}$ & $11.00 \mathrm{U}$ & & & $\mathrm{pCi} / \mathrm{L}$ \\
\hline & Technetium & $22.00 \mathrm{U}$ & $22.00 \mathrm{U}$ & & & $\mathrm{pCi} / \mathrm{L}$ \\
\hline & Uranium & $1.00 \mathrm{U}$ & 1.70 & 1.27 & 0.34 & $\mu \mathrm{g} / \mathrm{L}$ \\
\hline \multirow[t]{4}{*}{ BRCSW02 } & Gross Alpha & $6.00 \mathrm{U}$ & $11.00 \mathrm{U}$ & & & $\mathrm{pCi} / \mathrm{L}$ \\
\hline & Gross Beta & $8.00 \mathrm{U}$ & $11.00 \mathrm{U}$ & & & $\mathrm{pCi} / \mathrm{L}$ \\
\hline & Technetium & $22.00 \mathrm{U}$ & $22.00 \mathrm{U}$ & & & $\mathrm{pCi} / \mathrm{L}$ \\
\hline & Uranium & $1.00 \mathrm{U}$ & 1.90 & 1.47 & 0.38 & $\mu \mathrm{g} / \mathrm{L}$ \\
\hline \multirow[t]{4}{*}{ EDDSW01. } & Gross Alpha & $4.00 \mathrm{U}$ & $5.00 \mathrm{U}$ & & & $\mathrm{pCi} / \mathrm{L}$ \\
\hline & Gross Beta & $8.00 \mathrm{U}$ & $11.00 \mathrm{U}$ & & & $\mathrm{pCi} / \mathrm{L}$ \\
\hline & Technetium & $22.00 \mathrm{U}$ & $22.00 \mathrm{U}$ & & & $\mathrm{pCi} / \mathrm{L}$ \\
\hline & Uranium & $1.00 \mathrm{U}$ & 1.50 & 1.22 & 0.22 & $\mu \mathrm{g} / \mathrm{L}$ \\
\hline \multirow[t]{4}{*}{ LBCSW01 } & Gross Alpha & $4.00 \mathrm{U}$ & 7.00 & 5.50 & 1.29 & $\mathrm{pCi} / \mathrm{L}$ \\
\hline & Gross Beta & $8.00 \mathrm{U}$ & $11.00 \mathrm{U}$ & & & $\mathrm{pCi} / \mathrm{L}$ \\
\hline & Technetium & $22.00 \mathrm{U}$ & $22.00 \mathrm{U}$ & & & $\mathrm{pCi} / \mathrm{L}$ \\
\hline & Uranium & 1.10 & 1.60 & 1.40 & 0.22 & $\mu \mathrm{g} / \mathrm{L}$ \\
\hline \multirow[t]{4}{*}{ LBCSW02 } & Gross Alpha & $4.00 \mathrm{U}$ & 5.00 & 4.75 & 0.50 & $\mathrm{pCi} / \mathrm{L}$ \\
\hline & Gross Beta & $8.00 \mathrm{U}$ & $11.00 \mathrm{U}$ & & & $\mathrm{pCi} / \mathrm{L}$ \\
\hline & Technetium & $22.00 \mathrm{U}$ & $24.00 \mathrm{U}$ & & & $\mathrm{pCi} / \mathrm{L}$ \\
\hline & Uranium & 1.00 & 1.90 & 1.48 & 0.40 & $\mu \mathrm{g} / \mathrm{L}$ \\
\hline \multirow[t]{4}{*}{ LBCSW03 } & Gross Alpha & $4.00 \mathrm{U}$ & 10.00 & 6.75 & 2.50 & $\mathrm{pCi} / \mathrm{L}$ \\
\hline & Gross Beta & $8.00 \mathrm{U}$ & 11.00 & & & $\mathrm{pCi} / \mathrm{L}$ \\
\hline & Technetium & $22.00 \mathrm{U}$ & $22.00 \mathrm{U}$ & & & $\mathrm{pCi} / \mathrm{L}$ \\
\hline & Uranium & 1.10 & 2.70 & 1.85 & 0.70 & $\mu \mathrm{g} / \mathrm{L}$ \\
\hline \multirow[t]{4}{*}{ LBCSW04 } & Gross Alpha & 8.00 & 10.00 & 9.00 & 0.82 & $\mathrm{pCi} / \mathrm{L}$ \\
\hline & Gross Beta & 10.00 & 18.00 & 13.00 & 3.56 & $\mathrm{pCi} / \mathrm{L}$ \\
\hline & Technetium & $22.00 \mathrm{U}$ & $22.00 \mathrm{U}$ & & & $\mathrm{pCi} / \mathrm{L}$ \\
\hline & Uranium & 2.00 & 3.90 & 3.10 & 0.88 & $\mu \mathrm{g} / \mathrm{L}$ \\
\hline
\end{tabular}


Table 3.9. DOE/POATS groundwater monitoring for 1995: results for radionuclide parameters at surface water monitoring locations (continued)

\begin{tabular}{|c|c|c|c|c|c|c|}
\hline Well & Parameter & $\operatorname{Min}$ & Max & Mean & $\begin{array}{l}\text { Standard } \\
\text { Deviation }\end{array}$ & Units \\
\hline \multirow[t]{4}{*}{ NHPSW01 } & Gross Alpha & $5.00 \mathrm{U}$ & 8.00 & 6.25 & 1.50 & $\mathrm{pCi} / \mathrm{L}$ \\
\hline & Gross Beta & $8.00 \mathrm{U}$ & 9.00 & & & $\mathrm{pCi} / \mathrm{L}$ \\
\hline & Technetium & $22.00 \mathrm{U}$ & $22.00 \mathrm{U}$ & & & $\mathrm{pCi} / \mathrm{L}$ \\
\hline & Uranium & 4.10 & 8.70 & 7.10 & 2.08 & $\mu \mathrm{g} / \mathrm{L}$ \\
\hline \multirow[t]{4}{*}{ UNDSW01 } & Gross Alpha & $5.00 \mathrm{U}$ & $6.00 \mathrm{U}$ & & & $\mathrm{pC} / \mathrm{L}$ \\
\hline & Gross Beta & $8.00 \mathrm{U}$ & $11.00 \mathrm{U}$ & & & $\mathrm{pCi} / \mathrm{L}$ \\
\hline & Technetium & $22.00 \mathrm{U}$ & $22.00 \mathrm{U}$ & & & $\mathrm{pCi} / \mathrm{L}$ \\
\hline & Uranium & 1.90 & 2.10 & 2.00 & 0.08 & $\mu \mathrm{g} / \mathrm{L}$ \\
\hline \multirow[t]{4}{*}{ UNDSW02: } & Gross Alpha & $4.00 \mathrm{U}$ & $5.00 \mathrm{U}$ & & & $\mathrm{pCi} / \mathrm{L}$ \\
\hline & Gross Beta & $8.00 \mathrm{U}$ & $11.00 \mathrm{U}$ & & & $\mathrm{pCi} / \mathrm{L}$ \\
\hline & Technetium & $22.00 \mathrm{U}$ & $22.00 \mathrm{U}$ & & & $\mathrm{pCi} / \mathrm{L}$ \\
\hline & Uranium & 1.20 & 2.30 & 1.73 & 0.56 & $\mu \mathrm{g} / \mathrm{L}$ \\
\hline \multicolumn{2}{|c|}{ WDD-SW01 Gross Alpha } & $4.00 \mathrm{U}$ & 5.00 & & & $\mathrm{pCi} / \mathrm{L}$ \\
\hline & Gross Beta & $8.00 \mathrm{U}$ & $9.00 \mathrm{U}$ & & & $\mathrm{pCi} / \mathrm{L}$ \\
\hline & Technetium & $22.00 \mathrm{U}$ & $22,00 \mathrm{U}$ & & . & $\mathrm{pCi} / \mathrm{L}$ \\
\hline & Uranium & 2.10 & 3.60 & 2.78 & 0.61 & $\mu \mathrm{g} / \mathrm{L}$ \\
\hline \multicolumn{2}{|c|}{ WDD-SW02Gross Alpha } & $5.00 \mathrm{U}$ & $8.00 \mathrm{U}$ & & & $\mathrm{pCi} / \mathrm{L}$ \\
\hline & Gross Beta. & $8.00 \mathrm{U}$ & $12.00 \mathrm{U}$ & & & $\mathrm{pCi} / \mathrm{L}$ \\
\hline & Technetium & $22.00 \mathrm{U}$ & $22.00 \mathrm{U}$ & & & $\mathrm{pCi} / \mathrm{L}$ \\
\hline & Uranium & $1.00 \mathrm{U}$ & 3.40 & & & $\mu \mathrm{g} / \mathrm{L}$ \\
\hline \multicolumn{2}{|c|}{ WDD-SW03 Gross Alpha } & $4.00 \mathrm{U}$ & $5.00 \mathrm{U}$ & & & $\mathrm{pCi} / \mathrm{L}$ \\
\hline & Gross Beta & $8.00 \mathrm{U}$ & $9.00 \mathrm{U}$ & & & $\mathrm{pCi} / \mathrm{L}$ \\
\hline & Technetium & $22.00 \mathrm{U}$ & $22.00 \mathrm{U}$ & & & $\mathrm{pCi} / \mathrm{L}$ \\
\hline & Uranium & 2.20 & 5.20 & 3.50 & 1.12 & $\mu \mathrm{g} / \mathrm{L}$ \\
\hline
\end{tabular}

Data Qualifer (U)--Parameter not detected. Result is attainable detection limit for the sample. Mean and standard deviation functions not calculated if at least $50 \%$ of the results for the parameter were below detection. 
Table 3.10. DOE/PORTS groundwater monitoring for 1995:

results for volatile organic compounds at surface water monitoring locations

\begin{tabular}{|c|c|c|c|c|c|c|}
\hline Well & Parameter & Min & Max & Mean & $\begin{array}{l}\text { Standard } \\
\text { Deviation }\end{array}$ & Units \\
\hline \multirow[t]{2}{*}{ BRCSW01 } & 1,1,1-Trichloroethane & $2.00 \mathrm{U}$ & $2.00 \mathrm{U}$ & & & $\mu \mathrm{g} / \mathrm{L}$ \\
\hline & Trichloroethene & $2.00 \mathrm{U}$ & $2.00 \mathrm{U}$ & & & $\mu \mathrm{g} / \mathrm{L}$ \\
\hline \multirow[t]{2}{*}{ BRCSW02 } & 1,1,1-Trichloroethane & $2.00 \mathrm{U}$ & $2.00 \mathrm{U}$ & & & $\mu \mathrm{g} / \mathrm{L}$ \\
\hline & Trichloroethene & $2.00 \mathrm{U}$ & $2.00 \mathrm{U}$ & & & $\mu \mathrm{g} / \mathrm{L}$ \\
\hline \multirow{2}{*}{ EDDSW01 } & 1,1,1-Trichloroethane & $2.00 \mathrm{U}$ & $2.00 \mathrm{U}$ & & & $\mu \mathrm{g} / \mathrm{L}$ \\
\hline & Trichloroethene & $2.00 \mathrm{U}$ & $2.00 \mathrm{U}$ & & & $\mu \mathrm{g} / \mathrm{L}$ \\
\hline \multirow[t]{2}{*}{ LBCSW01 } & 1,1,1-Trichloroethane & $2.00 \mathrm{U}$ & $2.00 \mathrm{U}$ & & & $\mu \mathrm{g} / \mathrm{L}$ \\
\hline & Trichloroethene & $2.00 \mathrm{U}$ & $2.00 \mathrm{U}$ & & & $\mu \mathrm{g} / \mathrm{L}$ \\
\hline \multirow[t]{2}{*}{ LBCSW02 } & $1,1,1$-Trichloroethane & $2.00 \mathrm{U}$ & $2.00 \mathrm{U}$ & & & $\mu \mathrm{g} / \mathrm{L}$ \\
\hline & Trichloroethene & $2.00 \mathrm{U}$ & $2.00 \mathrm{U}$ & & & $\mu \mathrm{g} / \mathrm{L}$ \\
\hline \multirow[t]{2}{*}{ LBCSW03 } & 1,1,1-Trichloroethane & $2.00 \mathrm{U}$ & $2.00 \mathrm{U}$ & & & $\mu \mathrm{g} / \mathrm{L}$ \\
\hline & Trichloroethene & $2.00 \mathrm{U}$ & $2.00 \mathrm{U}$ & & & $\mu \mathrm{g} / \mathrm{L}$ \\
\hline \multirow[t]{2}{*}{ LBCSW04 } & 1,1,1-Trichloroethane & $2.00 \mathrm{U}$ & $2.00 \mathrm{U}$ & & & $\mu \mathrm{g} / \mathrm{L}$ \\
\hline & Trichloroethene & $2.00 \mathrm{U}$ & $2.00 \mathrm{U}$ & & & $\mu \mathrm{g} / \mathrm{L}$ \\
\hline \multirow[t]{2}{*}{ NHPSW01 } & 1,1,1-Trichloroethane & $2.00 \mathrm{U}$ & $2.00 \mathrm{U}$ & & & $\mu \mathrm{g} / \mathrm{L}$ \\
\hline & Trichloroethene & $2.00 \mathrm{U}$ & $2.00 \mathrm{U}$ & & & $\mu \mathrm{g} / \mathrm{L}$ \\
\hline \multirow[t]{2}{*}{ UNDSW01 } & 1,1,1-Trichloroethane & $2.00 \mathrm{U}$ & $2.00 \mathrm{U}$ & & & $\mu \mathrm{g} / \mathrm{L}$ \\
\hline & Trichloroethene & 3.00 & 5.00 & 4.00 & 0.82 & $\mu \mathrm{g} / \mathrm{L}$ \\
\hline \multirow[t]{2}{*}{ UNDSW02 } & 1,1,1-Trichloroethane & $2.00 \mathrm{U}$ & $2.00 \mathrm{U}$ & & & $\mu \mathrm{g} / \mathrm{L}$ \\
\hline & Trichloroethene & $2.00 \mathrm{U}$ & $2.00 \mathrm{U}$ & & & $\mu \mathrm{g} / \mathrm{L}$ \\
\hline \multicolumn{2}{|c|}{ WDD-SW01 1,1,1-Trichloroethane } & $2.00 \mathrm{U}$ & $2.00 \mathrm{U}$ & & & $\mu \mathrm{g} / \mathrm{L}$ \\
\hline & Trichloroethene & $2.00 \mathrm{U}$ & $2.00 \mathrm{U}$ & & & $\mu \mathrm{g} / \mathrm{L}$ \\
\hline \multicolumn{2}{|c|}{ WDD-SW021,1,1-Trichloroethane } & $2.00 \mathrm{U}$ & $2.00 \mathrm{U}$ & & & $\mu g / L$ \\
\hline & Trichloroethene & $2.00 \mathrm{U}$ & $2.00 \mathrm{U}$ & & & $\mu \mathrm{g} / \mathrm{L}$ \\
\hline WDD-SW03 & $1,1,1$-Trichloroethane & $2.00 \mathrm{U}$ & $2.00 \mathrm{U}$ & & & $\mu \mathrm{g} / \mathrm{L}$ \\
\hline & Trichloroethene & $2.00 \mathrm{U}$ & $2.00 \mathrm{U}$ & & & $\mu \mathrm{g} / \mathrm{L}$ \\
\hline
\end{tabular}

Data Qualifer (U)--Parameter not detected. Result is attainable detection limit for the sample. Mean and standard deviation functions not calculated if at least $50 \%$ of the results for the parameter were below detection. 
Table 3.11. DOE/PORTS groundwater monitoring for 1995: results for trichloroethene at X-701C

\begin{tabular}{|c|c|c|c|c|c|c|}
\hline Well & Parameter & Min & $\operatorname{Max}$ & Mean & $\begin{array}{l}\text { Standard } \\
\text { Deviation }\end{array}$ & Units \\
\hline \multicolumn{7}{|c|}{ Gallia Wells } \\
\hline$X 701-68 G$ & Trichloroethene & 44.00 & 47.00 & 45.50 & 2.12 & $\mu \mathrm{g} / \mathrm{L}$ \\
\hline$X 701-69 G$ & Trichloroethene & 1400.00 & 1800.00 & 1600.00 & 282.84 & $\mu \mathrm{g} / \mathrm{L}$ \\
\hline X701-70G & Trichloroethene & 250.00 & 280.00 & 272.50 & 15.00 & $\mu \mathrm{g} / \mathrm{L}$ \\
\hline
\end{tabular}

Data Qualifer (U)--Parameter not detected. Result is attainable detection limit for the sample. Mean and standard deviation functions not calculated if at least $50 \%$ of the results for the parameter were below detection. 
Table 3.12. DOE/POATS groundwater monitoring for 1995: results for radionuclide parameters at $X-701 \mathrm{C}$

\begin{tabular}{|c|c|c|c|c|c|c|}
\hline Well & Parameter & Min & Max & Mean & $\begin{array}{l}\text { Standard } \\
\text { Deviation }\end{array}$ & Units \\
\hline \multicolumn{7}{|c|}{ Gallia Wells } \\
\hline \multirow[t]{4}{*}{$X 701-68 G$} & Gross Alpha & $5.00 \mathrm{U}$ & $11.00 \mathrm{U}$ & & & $\mathrm{pCi} / \mathrm{L}$ \\
\hline & Gross Beta & 12.00 & 18.00 & 15.00 & 4.24 & $\mathrm{pCi} / \mathrm{L}$ \\
\hline & Technetium & $22.00 \mathrm{U}$ & 25.00 & & & $\mathrm{pCi} / \mathrm{L}$ \\
\hline & Uranium & $1.00 \mathrm{U}$ & $1.00 \mathrm{U}$ & & & $\mu \mathrm{g} / \mathrm{L}$ \\
\hline \multirow[t]{4}{*}{$X 701-69 G$} & Gross Alpha & $11.00 \mathrm{U}$ & $17.00 \mathrm{U}$ & & & $\mathrm{pCi} / \mathrm{L}$ \\
\hline & Gross Beta & $17.00 \mathrm{U}$ & 21.00 & & & $\mathrm{pCi} / \mathrm{L}$ \\
\hline & Technetium & $22.00 \mathrm{U}$ & $22.00 \mathrm{U}$ & & & $\mathrm{pCi} / \mathrm{L}$ \\
\hline & Uranium & 5.20 & 6.10 & 5.65 & 0.64 & $\mu \mathrm{g} / \mathrm{L}$ \\
\hline \multirow[t]{4}{*}{$\mathrm{X} 701-70 \mathrm{G}$} & Gross Alpha & $5.00 \mathrm{U}$ & $6.00 \mathrm{U}$ & & & $\mathrm{pCi} / \mathrm{L}$ \\
\hline & Gross Beta & 35.00 & 43.00 & 39.00 & 5.66 & $\mathrm{pCi} / \mathrm{L}$ \\
\hline & Technetium & 42.00 & 43.00 & 42.50 & 0.71 & $\mathrm{pCi} / \mathrm{L}$ \\
\hline & Uranium & 2.70 & 3.00 & 2.85 & 0.21 & $\mu \mathrm{g} / \mathrm{L}$ \\
\hline
\end{tabular}

Data Qualifer (U)--Parameter not detected. Result is attainable detection limit for the sample. Mean and standard deviation functions not calculated if at least $50 \%$ of the results for the parameter
were below detection. 
Table 3.13. DOE/PORTS groundwater monitoring for 1995: results for radionuclide parameters at $X-735$

\begin{tabular}{|c|c|c|c|c|c|c|}
\hline Well & Parameter & Min & Max & Mean & $\begin{array}{l}\text { Standard } \\
\text { Deviation }\end{array}$ & Units \\
\hline \multicolumn{7}{|c|}{ Berea Wells } \\
\hline \multirow[t]{4}{*}{$X 735-16 B$} & Gross Alpha & $4.00 \mathrm{U}$ & $4.00 \mathrm{U}$ & & & $\mathrm{pCi} / \mathrm{L}$ \\
\hline & Gross Beta & $11.00 \mathrm{U}$ & $11.00 \mathrm{U}$ & & & $\mathrm{pCi} / \mathrm{L}$ \\
\hline & Technetium & $21.00 \mathrm{U}$ & $21.00 \mathrm{U}$ & & & $\mathrm{pCi} / \mathrm{L}$ \\
\hline & Uranium & $1.00 \mathrm{U}$ & $1.00 \mathrm{U}$ & & & $\mu \mathrm{g} / \mathrm{L}$ \\
\hline \multirow[t]{4}{*}{ X735-17B } & Gross Alpha & $5.00 \mathrm{U}$ & $5.00 \mathrm{U}$ & & & $\mathrm{pCi} / \mathrm{L}$ \\
\hline & Gross Beta & $11.00 \mathrm{U}$ & $11.00 \mathrm{U}$ & & & $\mathrm{pCi} / \mathrm{L}$ \\
\hline & Technetium & $24.00 \mathrm{U}$ & $24.00 \mathrm{U}$ & & & $\mathrm{pCi} / \mathrm{L}$ \\
\hline & Uranium & 190 & 1.90 & 1.90 & & $\mu \mathrm{g} / \mathrm{L}$ \\
\hline \multirow[t]{4}{*}{ X735-18B } & Gross Alpha & 17.00 & 17.00 & 17.00 & & $\mathrm{pCi} / \mathrm{L}$ \\
\hline & Gross Beta & 16.00 & 16.00 & 16.00 & & $\mathrm{pCi} / \mathrm{L}$ \\
\hline & Technetium & $24.00 \mathrm{U}$ & $24.00 \mathrm{U}$ & & & $\mathrm{pCi} / \mathrm{L}$ \\
\hline & Uranium & 3.30 & 3.30 & 3.30 & & $\mu \mathrm{g} / \mathrm{L}$ \\
\hline \multirow[t]{4}{*}{$X 735-20 B$} & Gross Alpha & 15.00 & 15.00 & 15.00 & & $\mathrm{pCi} / \mathrm{L}$ \\
\hline & Gross Beta & 18.00 & 18.00 & 18.00 & & $\mathrm{pCi} / \mathrm{L}$ \\
\hline & Technetium & $24.00 \mathrm{U}$ & $24.00 \mathrm{U}$ & & & $\mathrm{pCi} / \mathrm{L}$ \\
\hline & Uranium & 9.50 & 9.50 & 9.50 & & $\mu \mathrm{g} / \mathrm{L}$ \\
\hline \multicolumn{7}{|c|}{ Gallia Wells } \\
\hline X735-01GA & Gross Alpha & $4.00 \mathrm{U}$ & $4.00 \mathrm{U}$ & & & $\mathrm{pCi} / \mathrm{L}$ \\
\hline & Gross Beta & $11.00 \mathrm{U}$ & $11.00 \mathrm{U}$ & & & $\mathrm{pCi} / \mathrm{L}$ \\
\hline & Technetium & $22.00 \mathrm{U}$ & $24.00 \mathrm{U}$ & & & $\mathrm{pCi} / \mathrm{L}$ \\
\hline & Uranium & $1.00 \mathrm{U}$ & $1.00 \mathrm{U}$ & & & $\mu \mathrm{g} / \mathrm{L}$ \\
\hline X735-02GA & Gross Alpha & $3.00 \mathrm{U}$ & $4.00 \mathrm{U}$ & & & $\mathrm{pCi} / \mathrm{L}$ \\
\hline & Gross Beta & $11.00 \mathrm{U}$ & $11.00 \mathrm{U}$ & & & $\mathrm{pCi} / \mathrm{L}$ \\
\hline & Technetium & $22.00 \mathrm{U}$ & $24.00 \mathrm{U}$ & & & $\mathrm{pCi} / \mathrm{L}$ \\
\hline & Uranium & $1.00 \mathrm{U}$ & $1.00 \mathrm{U}$ & & & $\mu \mathrm{g} / \mathrm{L}$ \\
\hline X735-03GA & Gross Alpha & $4.00 \mathrm{U}$ & $4.00 \mathrm{U}$ & & & $\mathrm{pC} / \mathrm{L}$ \\
\hline & Gross Beta & $11.00 \mathrm{U}$ & $11.00 \mathrm{U}$ & & & $\mathrm{pCi} / \mathrm{L}$ \\
\hline & Technetium & $22.00 \mathrm{U}$ & $24.00 \mathrm{U}$ & & & $\mathrm{pCi} / \mathrm{L}$ \\
\hline
\end{tabular}


Table 3.13. DOE/PORTS groundwater monitoring for 1995: results for radionuclide parameters at $\mathbf{X}-735$ (continued)

\begin{tabular}{|c|c|c|c|c|c|c|}
\hline Well & Parameter & Min & Max & Mean & $\begin{array}{l}\text { Standard } \\
\text { Deviation }\end{array}$ & Units \\
\hline \multicolumn{7}{|c|}{ Gallia Wells (cont) } \\
\hline X735-03GA & Uranium & $1.00 \mathrm{U}$ & $1.00 \mathrm{U}$ & & & $\mu \mathrm{g} / \mathrm{L}$ \\
\hline \multirow[t]{4}{*}{$\mathrm{X} 735-04 \mathrm{GA}$} & Gross Alpha & $3.00 \mathrm{U}$ & $4.00 \mathrm{U}$ & & & $\mathrm{pCi} / \mathrm{L}$ \\
\hline & Gross Beta & $11.00 \mathrm{U}$ & $11.00 \mathrm{U}$ & & & $\mathrm{pCi} / \mathrm{L}$ \\
\hline & Technetium & $22.00 \mathrm{U}$ & $24.00 \mathrm{U}$ & & & $\mathrm{pCi} / \mathrm{L}$ \\
\hline & Uranium & $1.00 \mathrm{U}$ & $1.00 \mathrm{U}$ & & & $\mu \mathrm{g} / \mathrm{L}$ \\
\hline \multirow[t]{4}{*}{$\mathrm{X} 735-05 \mathrm{GA}$} & Gross Alpha & $4.00 \mathrm{U}$ & $5.00 \mathrm{U}$ & & & $\mathrm{pCi} / \mathrm{L}$ \\
\hline & Gross Beta & $11.00 \mathrm{U}$ & $11.00 \mathrm{U}$ & & & $\mathrm{pCi} / \mathrm{L}$ \\
\hline & Technetium & $22.00 \mathrm{U}$ & $24.00 \mathrm{U}$ & & & $\mathrm{pCi} / \mathrm{L}$ \\
\hline & Uranium & $1.00 \mathrm{U}$ & $1.00 \mathrm{U}$ & & & $\mu \mathrm{g} / \mathrm{L}$ \\
\hline \multirow[t]{4}{*}{ X735-06GA } & Gross Alpha & 4.00 & 5.00 & 4.50 & 0.71 & $\mathrm{pC} \mathrm{i} / \mathrm{L}$ \\
\hline & Gross Beta & $11.00 \mathrm{U}$ & 17.00 & & & $\mathrm{pCi} / \mathrm{L}$ \\
\hline & Technetium & $22.00 \mathrm{U}$ & 25.00 & & & $\mathrm{pCi} / \mathrm{L}$ \\
\hline & Uranium & $1.00 \mathrm{U}$ & $1.00 \mathrm{U}$ & & & $\mu \mathrm{g} / \mathrm{L}$ \\
\hline \multirow[t]{4}{*}{ X735-13GA } & Gross Alpha & 5.00 & 12.00 & 8.50 & 4.95 & $\mathrm{pCi} / \mathrm{L}$ \\
\hline & Gross Beta & $11.00 \mathrm{U}$ & 15.00 & & & $\mathrm{pCi} / \mathrm{L}$ \\
\hline & Technetium & $22.00 \mathrm{U}$ & $24.00 \mathrm{U}$ & & & $\mathrm{pCi} / \mathrm{L}$ \\
\hline & Uranium & 4.90 & 9.20 & 7.05 & 3.04 & $\mu \mathrm{g} / \mathrm{L}$ \\
\hline \multirow[t]{4}{*}{$\mathrm{X} 735-19 \mathrm{G}$} & Gross Alpha & 13.00 & 13.00 & 13.00 & & $\mathrm{pCi} / \mathrm{L}$ \\
\hline & Gross Beta & 13.00 & 13.00 & 13.00 & & $\mathrm{pCi} / \mathrm{L}$ \\
\hline & Technetium & $24.00 \mathrm{U}$ & $24.00 \mathrm{U}$ & & & $\mathrm{pCi} / \mathrm{L}$ \\
\hline & Uranium & 18.80 & 18.80 & 18.80 & & $\mu \mathrm{g} / \mathrm{L}$ \\
\hline \multirow[t]{4}{*}{$\mathrm{X} 735-21 \mathrm{G}$} & Gross Alpha & 22.00 & 22.00 & 22.00 & & $\mathrm{pCi} / \mathrm{L}$ \\
\hline & Gross Beta & 14.00 & 14.00 & 14.00 & & $\mathrm{pCi} / \mathrm{L}$ \\
\hline & Technetium & $24.00 \mathrm{U}$ & $24.00 \mathrm{U}$ & & & $\mathrm{pCi} / \mathrm{L}$ \\
\hline & Uranium & 5.60 & 5.60 & 5.60 & & $\mu \mathrm{g} / \mathrm{L}$ \\
\hline
\end{tabular}

Data Qualifer (U)--Parameter not detected. Result is attainable detection limit for the sample. Mean and standard deviation functions not calculated if at least $50 \%$ of the results for the parameter were below detection. 
Table 3.14. DOE/PORTS groundwater monitoring for 1995: results for trichloroethene at X-735

\begin{tabular}{|c|c|c|c|c|c|c|}
\hline Well & Parameter & Min & $\operatorname{Max}$ & Mean & $\begin{array}{l}\text { Standard } \\
\text { Deviation }\end{array}$ & Units \\
\hline \multicolumn{7}{|c|}{ Berea Wells } \\
\hline$X 735-16 B$ & Trichloroethene & $2.00 \mathrm{U}$ & $2.00 \mathrm{U}$ & & & $\mu \mathrm{g} / \mathrm{L}$ \\
\hline X735-17B & Trichloroethene & $2.00 \mathrm{U}$ & $2.00 \mathrm{U}$ & & & $\mu \mathrm{g} / \mathrm{L}$ \\
\hline $\mathrm{X} 735-18 \mathrm{~B}$ & Trichloroethene & $2.00 \mathrm{U}$ & $2.00 \mathrm{U}$ & & & $\mu \mathrm{g} / \mathrm{L}$ \\
\hline$X 735-20 B$ & Trichloroethene & $2.00 \mathrm{U}$ & $2.00 \mathrm{U}$ & & & $\mu \mathrm{g} / \mathrm{L}$ \\
\hline \multicolumn{7}{|c|}{ Gallia Wells } \\
\hline $\mathrm{X} 735-01 \mathrm{GA}$ & Trichloroethene & $2.00 \mathrm{U}$ & $2.00 \mathrm{U}$ & & & $\mu \mathrm{g} / \mathrm{L}$ \\
\hline $\mathrm{X} 735-02 \mathrm{GA}$ & Trichloroethene & $2.00 \mathrm{U}$ & $2.00 \mathrm{U}$ & & & $\mu \mathrm{g} / \mathrm{L}$ \\
\hline $\mathrm{X} 735-03 \mathrm{GA}$ & Trichloroethene & $2.00 \mathrm{U}$ & $2.00 \mathrm{U}$ & & & $\mu \mathrm{g} / \mathrm{L}$ \\
\hline X735-04GA & Trichloroethene & $2.00 \mathrm{U}$ & $2.00 \mathrm{U}$ & & & $\mu \mathrm{g} / \mathrm{L}$ \\
\hline $\mathrm{X} 735-05 \mathrm{GA}$ & Trichloroethene & $2.00 \mathrm{U}$ & $2.00 \mathrm{U}$ & & & $\mu \mathrm{g} / \mathrm{L}$ \\
\hline X735-06GA & Trichloroethene & $2.00 \mathrm{U}$ & $2.00 \mathrm{U}$ & & & $\mu \mathrm{g} / \mathrm{L}$ \\
\hline X735-13GA & Trichloroethene & $2.00 \mathrm{U}$ & $2.00 \mathrm{U}$ & & & $\mu \mathrm{g} / \mathrm{L}$ \\
\hline $\mathrm{X} 735-19 \mathrm{G}$ & Trichloroethene & $2.00 \mathrm{U}$ & $2.00 \mathrm{U}$ & & & $\mu \mathrm{g} / \mathrm{L}$ \\
\hline$X 735-21 G$ & Trichloroethene & $2.00 \mathrm{U}$ & $2,00 \mathrm{U}$ & & & $\mu g / L$ \\
\hline
\end{tabular}

Data Qualifer (U)--Parameter not detected. Result is attainable detection limit for the sample. Mean and standard deviation functions not calculated if at least $50 \%$ of the results for the parameter were below detection. 